Bull. Soc. math. France

130 (3), 2002, p. 349-386

\title{
MASSE DES POINTES, TEMPS DE RETOUR ET ENROULEMENTS EN COURBURE NÉGATIVE
}

\author{
par Nathanä̈l Enriquez \& JaCques Franchi
}

\begin{abstract}
RÉSUmÉ. - Soient $\Gamma$ un groupe discret géométriquement fini d'isométries d'une variété de Hadamard pincée $X$ et $\mathcal{P}$ une pointe de l'orbifold associé $\mathcal{M}:=\Gamma \backslash X$. Munissant $T^{1} \mathcal{M}$ de sa mesure de Patterson-Sullivan $m$, nous obtenons une estimation asymptotique de la masse d'un petit voisinage horocyclique de $\mathcal{P}$, moyennant une hypothèse sur la croissance du sous-groupe parabolique associé à $\mathcal{P}$, hypothèse qui est réalisée si $X$ est symétrique de rang 1 . Nous en déduisons une estimation asymptotique du temps de retour du flot géodésique près de $\mathcal{P}$, et de la loi de la durée d'une excursion du flot géodésique près de $\mathcal{P}$.

Dans le cas particulier des orbifolds hyperboliques réels ou complexes, nous précisons ces estimations, et nous calculons de plus la loi asymptotique de l'enroulement d'une excursion du flot géodésique près de $\mathcal{P}$.
\end{abstract}

Texte reçu le 22 mai 2001, accepté le 16 janvier 2002

NathanaËl Enriquez, Laboratoire de Probabilités de Paris 6, 4place Jussieu, tour 56, 3ème étage, 75252 Paris Cedex 05 • E-mail : enriquez@ccr.jussieu.fr

JaCQUes Franchi, I.R.M.A., Université Louis Pasteur, 7 rue René Descartes, 67084 Strasbourg Cedex • E-mail : franchi@math.u-strasbg.fr

Classification mathématique par sujets (2000). — 37A50, 37D40.

Mots clefs. - Variété hyperbolique de volume infini, mesure de Patterson-Sullivan, mesure de Palm. 


\begin{abstract}
Mass of cusps, time of return and windings in negative curvature)
Let $\Gamma$ be a geometrically finite discrete group of isometries of a Hadamard manifold $X$, and $\mathcal{P}$ a cusp of the orbifold associated to $\mathcal{M}:=\Gamma \backslash X . T^{1} \mathcal{M}$ being endowed with its Bowen-Margulis-Patterson-Sullivan $m$, we obtain the asymptotic of the mass of a small horocyclic neighbourhood of $\mathcal{P}$, after introducing an assumption on the growth of the parabolic neighbourhood associated with $\mathcal{P}$, which is automatically verified when $X$ is symmetric of rank 1 . We deduce the asymptotic of the time of return of the geodesic flow near $\mathcal{P}$.

In the special case of real and complex hyperbolic orbifolds, we precise these asymptotics, and we compute also the asmptotic law of the winding of the geodesic flow near $\mathcal{P}$.
\end{abstract}

\title{
1. Introduction
}

Les variétés hyperboliques et leur flot géodésique constituent un exemple fondamental de système dynamique. En courbure constante et volume fini, la mesure invariante associée est naturellement la mesure de Liouville. Son substitut naturel en volume infini ou en courbure variable pincée, au moins dans le cas géométriquement fini, est la mesure $m$ utilisée en particulier par Bowen, Margulis et Sullivan [17], [18], qui est construite sur le fibré tangent unitaire à partir de la famille des mesures $\mu_{z}$ sur l'ensemble-limite de la variété, famille construite originellement par Patterson [15] dans le cadre de la courbure constante. Ces mesures apparaissent plus tard dans le cadre de la courbure variable, dans [14]. Voir aussi parmi les travaux plus récents [2] et [5].

Cette mesure $m$, de Patterson-Sullivan, présente donc un grand intérêt. Elle est bien sûr invariante par le flot géodésique, mais elle est très singulière, et donc délicate à étudier.

Nous nous proposons ici d'abord de calculer la masse asymptotique sous $m$ d'un petit voisinage horocyclique d'une pointe $\mathcal{P}$ d'un orbifold $\mathcal{M}$, et d'en déduire l'asymptotique sous la mesure invariante $m$ des temps de retour du flot géodésique sur un petit horocycle de la pointe $\mathcal{P}$, via la masse de la mesure de Palm induite sur ce petit horocycle par $m$. Notons que cette dernière masse fournit également la queue de la loi de la profondeur de pénétration d'une excursion du flot géodésique près de $\mathcal{P}$.

Nous évaluons aussi la loi asymptotique sous $m$ de la durée d'une telle excursion.

Nous effectuons ces calculs dans le cadre général d'un orbifold géométriquement fini de courbure négative pincée, avec une hypothèse additionnelle sur la croissance du groupe d'holonomie de la pointe $\mathcal{P}$, hypothèse qui est réalisée au moins dans le cas des espaces symétriques de rang 1.

Observant que les trois quantités évoquées ci-dessus s'obtiennent par l'évaluation de séries rappelant les séries de Poincaré, nous sommes naturellement

TOME $130-2002-\mathrm{N}^{\mathrm{O}} 3$ 
conduits à regrouper leur calcul sous la forme de l'étude générale de telles séries, que nous nommons « séries de Poincaré généralisées ». Nous appliquons ensuite cette étude générale aux trois quantités évoquées ci-dessus.

$\mathrm{Du}$ fait de la variation de la courbure, il n'est pas possible de donner un équivalent exact des quantités qui nous intéressent ici, et donc nos résultats dans le cadre général donnent un exposant précis, mais ne peuvent au niveau des constantes fournir qu'un encadrement.

C'est pourquoi nous focalisons ensuite notre étude sur deux cas particuliers fondamentaux, celui de la courbure constante, c'est-à-dire celui des orbifolds hyperboliques réels, et celui des orbifolds hyperboliques complexes. Nous précisons alors nos calculs précédents, et obtenons des équivalents exacts pour les quantités considérées, fournissant explicitement toutes les constantes.

Dans ces deux cas réel et complexe, nous calculons de plus la loi asymptotique sous $m$ des enroulements du flot géodésique au cours d'une excursion près de $\mathcal{P}$.

Ce calcul est à mettre en perspective avec les travaux précédents des auteurs avec Y. Le Jan dans le cas réel de la courbure négative constante [7], où il s'agissait de calculer, au moyen du calcul stochastique, l'enroulement asymptotique global d'une géodésique. Un travail antérieur de Guivarc'h et Le Jan [10], qui traitait le cas particulier du quotient par le groupe modulaire, montre le lien de ce sujet avec les problèmes d'approximations diophantiennes. Dans le présent travail, nous décrivons la loi de l'enroulement des excursions géodésiques individuelles sous leur mesure de Palm (relative à $m$ ).

Les auteurs remercient vivement Frédéric Paulin pour son aide obligeante concernant le passage de la courbure négative constante à la courbure négative pincée.

\section{Notations et rappels}

Sauf mention contraire, les rappels se réfèrent à [2].

Soit $X$ une variété riemannienne complète simplement connexe à courbure $\kappa$ négative pincée : $-\infty<-\alpha^{2} \leq \kappa \leq-1$.

Notons $d$ sa distance riemannienne, $\partial X$ son bord à l'infini, et $d_{x}$ la distance visuelle vue de $x$, définie sur $\partial X$ par : pour tous $x$ dans $X$ et $a, b$ dans $\partial X$

$$
d_{x}(a, b):=\exp \left(-\frac{1}{2} \lim _{t \rightarrow \infty}\left(d\left(a_{t}, x\right)+d\left(b_{t}, x\right)-d\left(a_{t}, b_{t}\right)\right)\right),
$$

où $a_{t}, b_{t}$ parcourent n'importe quels rayons géodésiques tendant vers $a, b$ respectivement.

Pour tout $a \in \partial X$, la fonction (distance horocyclique) de Busemann $\beta_{a}$ est définie sur $X^{2}$ par

$$
\beta_{a}(x, y):=\lim _{t \rightarrow \infty}\left(d\left(a_{t}, x\right)-d\left(a_{t}, y\right)\right)
$$

BULLETIN DE LA SOCIÉtÉ MATHÉMATIQUE DE FRANCE 
pour $a_{t}$ tendant vers $a$ lorsque $t \rightarrow \infty$, le long de n'importe quel rayon géodésique.

Soit $\Gamma$ un groupe discret (non élémentaire) géométriquement fini d'isométries de $X$ (voir [3]), d'ensemble limite noté $\Lambda(\Gamma)$ et d'exposant critique noté $\delta$ (égal à la dimension de Hausdorff de $\Lambda(\Gamma)$ pour toute $\left.d_{x}\right)$.

Notons $\left\{\mu_{x} \mid x \in X\right\}$ la famille des mesures de Patterson, qui sont finies, supportées par $\Lambda(\Gamma)$ et vérifient

$$
\frac{\mathrm{d} \mu_{x}}{\mathrm{~d} \mu_{y}}(a)=\mathrm{e}^{-\delta \beta_{a}(x, y)} \quad \text { pour tous } x, y \in X \text { et } a \in \partial X,
$$

ainsi que

$$
\mu_{x} \circ \gamma^{-1}=\mu_{\gamma x} \quad \text { pour tous } x \in X \text { et } \gamma \in \Gamma .
$$

Fixons un point de référence $x_{0} \in X$.

À tout vecteur unitaire tangent $v \in T^{1} X$, associons les extrémités répulsive $v_{-}$et attractive $v_{+}$de la géodésique orientée qu'il engendre, ainsi que la distance algébrique $s$ entre la projection orthogonale de $x_{0}$ sur cette géodésique et la base $\pi(v) \in X$ de $v$. L'application de $T^{1} X$ dans $\partial X \times \partial X \times \mathbb{R}$ qui à $v$ associe $\left(v_{-}, v_{+}, s\right)$ est un homéomorphisme.

Notons $\tilde{m}$ la mesure de Patterson-Sullivan sur $T^{1} X$, définie par

$$
\mathrm{d} \tilde{m}(v)=\mathrm{d} \tilde{m}\left(v_{-}, v_{+}, s\right):=\mathrm{d}_{x_{0}}\left(v_{-}, v_{+}\right)^{-2 \delta} \mathrm{d} \mu_{x_{0}}\left(v_{-}\right) \mathrm{d} \mu_{x_{0}}\left(v_{+}\right) \mathrm{d} s .
$$

Elle ne dépend pas de $x_{0}$, est invariante par le flot géodésique, et est $\Gamma$-invariante.

Elle induit donc une mesure $m$ invariante par le flot géodésique sur $T^{1} \mathcal{M}$, où $\mathcal{M}$ est l'orbifold riemannien $\Gamma \backslash X$.

On montre que le flot géodésique est $m$-ergodique.

Faisons l'hypothèse que $X$ admet au moins un point fixe parabolique $\xi \in \partial X$, et notons $\Gamma_{\xi}$ le stabilisateur de $\xi$ dans $\Gamma$, puis $\delta_{\xi}$ l'exposant critique de $\Gamma_{\xi}$.

Rappelons que lorsque $X$ est un espace hyperbolique réel, on a $\delta_{\xi}=\frac{1}{2} k$, où $k$ est le rang du sous-groupe parabolique $\Gamma_{\xi}$.

Supposons de plus que $\delta>\delta_{\xi}$, ce qui est automatique dans le cas des espaces symétriques de rang 1, mais n'est pas toujours vrai en courbure variable (voir [5]). Cette hypothèse assure que les mesures de Patterson n'ont pas d'atome en $\xi$ (voir [5]), et aussi, que la pointe est de $m$-mesure finie.

Notons $\mathcal{P}$ la pointe de $\mathcal{M}$ associée à $\xi$, c'est-à-dire le bout du quotient par $\Gamma$ de l'enveloppe convexe de $\Lambda(\Gamma)$ dans $X$ correspondant à la classe de conjugaison de $\Gamma_{\xi}$ dans $\Gamma$.

Soit $\left\{B_{h} \mid h \in \mathbb{R}\right\}$ l'unique famille d'horoboules ouvertes basées en $\xi$ vérifiant:

a) $B_{h}$ converge vers $\xi$ dans $X \cup \partial X$ lorsque $h \rightarrow+\infty$;

b) $d\left(H_{h}, H_{h^{\prime}}\right)=\left|h-h^{\prime}\right|$, où $H_{h}:=\partial B_{h}$;

TOME $130-2002-\mathrm{N}^{\mathrm{O}} 3$ 
c) $\mathcal{P}_{h}:=\Gamma_{\xi} \backslash B_{h}$ s'injecte dans $\mathcal{M}$ par la projection canonique $\Gamma_{\xi} \backslash X \rightarrow \Gamma \backslash X$ si et seulement si $h \geq 0$.

On dit que $\mathcal{P}_{h}$ est le voisinage de Margulis canonique de hauteur $h$ de la pointe $\mathcal{P}$.

Notons $\pi_{\xi}$ l'homéomorphisme $\Gamma_{\xi}$-équivariant de $\partial X \backslash\{\xi\}$ sur $H_{0}$ qui à $a$ associe l'unique point d'intersection de l'horosphère $H_{0}$ avec la géodésique reliant $a$ et $\xi$.

Soit $d_{\xi}$ la distance de Hamenstädt sur $\partial X \backslash\{\xi\}$, invariante par $\Gamma_{\xi}$, définie (voir [12, Appendix]) par

$$
d_{\xi}(a, b):=\lim _{t \rightarrow+\infty} \mathrm{e}^{t} d_{\xi_{t}}(a, b),
$$

où $\xi_{t}$ est n'importe quel rayon géodésique partant sur $H_{0}$ et arrivant en $\xi$.

Lorsque $X$ est le demi-espace de Poincaré de dimension $N+1$ et $\xi=\infty$, on vérifie que $d_{\xi}$ est un multiple de la distance euclidienne sur $\partial X \backslash\{\xi\} \equiv \mathbb{R}^{N}$.

Nous aurons besoin (voir $[2,1.3]$ ) de la variante suivante de l'inégalité CAT $(-1)$, relative au cas où l'un des points du triangle est situé à l'infini.

Lemme 1. - Soient $a \in \partial X, x, y \in X, \alpha$ sur le rayon géodésique $[x, a[$ et $\beta \in\left[y, a\left[\right.\right.$, et soient $\bar{a}, \bar{x}, \bar{y}, \bar{\alpha}, \bar{\beta}$ des homologues dans $\mathbb{H}^{2}: \bar{a} \in \partial \mathbb{H}^{2}, \bar{x}, \bar{y} \in \mathbb{H}^{2}$, $\bar{\alpha} \in[\bar{x}, \bar{a}[, \bar{\beta} \in[\bar{y}, \bar{a}[$, tels que

$$
d(x, y)=d(\bar{x}, \bar{y}), \quad d(x, \alpha)=d(\bar{x}, \bar{\alpha}), \quad d(y, \beta)=d(\bar{y}, \bar{\beta}) .
$$

Alors on a $d(\alpha, \beta) \leq d(\bar{\alpha}, \bar{\beta})$.

Démonstration. - Notons $a_{s}$ le point de $\left[x, a\left[\right.\right.$ situé à distance $s$ de $x$, et $\beta_{s}$ le point de $\left[y, a_{s}\right]$ situé à distance $d(y, \beta)$ de $y$, puis $\bar{a}_{s}, \bar{\beta}_{s}$ les points homologues dans $\mathbb{H}^{2}$.

L'inégalité $\mathrm{CAT}(-1)$ appliquée au triangle $\left(a_{s}, x, y\right)$ donne $d\left(\alpha, \beta_{s}\right) \leq$ $d\left(\bar{\alpha}, \bar{\beta}_{s}\right)$, et le théorème de comparaison de Topogonov (voir $[3,1.1 .3]$ ) assure que les angles $\left(\beta_{s}, y, \beta\right)$ et $\left(\bar{\beta}_{s}, \bar{y}, \bar{\beta}\right)$ tendent vers 0 lorsque $s \rightarrow \infty$, et donc que $d\left(\beta_{s}, \beta\right)$ et $d\left(\bar{\beta}_{s}, \bar{\beta}\right)$ tendent vers 0 lorsque $s \rightarrow \infty$; d'où le résultat en passant à la limite dans l'inégalité ci-dessus.

\section{Séries de Poincaré généralisées}

Pour $\xi_{t}$ rayon géodésique partant sur $H_{0}$ et arrivant en $\xi$ comme ci-dessus, la mesure $\mathrm{e}^{\delta t} \mu_{\xi_{t}}$ converge lorsque $t \rightarrow+\infty$ vers une mesure $\mu_{\xi}$ supportée par $\Lambda(\Gamma)$, invariante par $\Gamma_{\xi}$ et vérifiant :

$$
\mathrm{d} \mu_{\xi}(a)=\mathrm{e}^{-\delta \beta_{a}\left(\pi_{\xi}(a), x\right)} \mathrm{d} \mu_{x}(a) \text { pour tout } x \in X .
$$

BULLETIN DE LA SOCIÉtÉ MATHÉMATIQUE DE FRANCE 
En effet, (3) et la propriété de cocycle de la fonction $\beta_{a}$ entraînent que

$$
\begin{aligned}
\frac{\mathrm{d} \mu_{\xi_{t}}}{\mathrm{~d} \mu_{x}}(a)=\mathrm{e}^{-\delta \beta_{a}\left(\xi_{t}, x\right)} & =\mathrm{e}^{-\delta\left(\beta_{a}\left(\xi_{t}, \xi_{t}^{\prime}\right)+\beta_{a}\left(\xi_{t}^{\prime}, \pi_{\xi}(a)\right)+\beta_{a}\left(\pi_{\xi}(a), x\right)\right)} \\
& =\mathrm{e}^{\mathcal{O}\left(d\left(\xi_{t}, \xi_{t}^{\prime}\right)\right)-\delta t-\delta \beta_{a}\left(\pi_{\xi}(a), x\right)},
\end{aligned}
$$

où $\xi_{t}^{\prime}$ désigne l'intersection de $H_{t}$ avec la géodésique reliant $a$ et $\xi$.

Par invariance, $\mu_{\xi}$ induit une mesure $\mu_{\mathcal{P}}$ sur $\Gamma_{\xi} \backslash(\Lambda(\Gamma) \backslash\{\xi\})$, qui est relativement compact puisque $\Gamma$ est géométriquement fini.

Notons $\lambda_{\mathcal{P}}$ la masse de $\mu_{\mathcal{P}}$, qui ne dépend que de l'orbite de $\xi$ sous $\Gamma$, et donc que de la pointe $\mathcal{P}$.

Pour tout $v \in T^{1} X$ engendrant une géodésique sans extrémité en $\xi$, comme on a pour tout $t$ :

$$
\mathrm{d} \tilde{m}(v)=\left(\mathrm{e}^{t} \mathrm{~d}_{\xi_{t}}\left(v_{-}, v_{+}\right)\right)^{-2 \delta} \mathrm{e}^{\delta t} \mathrm{~d} \mu_{\xi_{t}}\left(v_{-}\right) \mathrm{e}^{\delta t} \mathrm{~d} \mu_{\xi_{t}}\left(v_{+}\right) \mathrm{d} s,
$$

on obtient via $t \rightarrow+\infty$ :

$$
\mathrm{d} \tilde{m}(v)=\mathrm{d}_{\xi}\left(v_{-}, v_{+}\right)^{-2 \delta} \mathrm{d} \mu_{\xi}\left(v_{-}\right) \mathrm{d} \mu_{\xi}\left(v_{+}\right) \mathrm{d} s .
$$

Fixons un domaine fondamental $\mathcal{D}$ pour l'action de $\Gamma_{\xi}$ sur $\Lambda(\Gamma)$, mesurable et relativement compact dans $\Lambda(\Gamma) \backslash\{\xi\}$.

Pour $a \neq b$ dans $\partial X \backslash\{\xi\}$ et $h>0$, notons $\ell_{h}(a, b)$ la longueur du segment géodésique intersection de la géodésique reliant $a$ et $b$ avec l'horoboule $B_{h}$, bien sûr nulle si cette intersection est vide.

Le lemme suivant fournit deux formules d'approximation essentielles pour évaluer ensuite les séries de Poincaré généralisées.

LEMme 2. - Nous avons uniformément par rapport à $a, b \in \partial X \backslash\{\xi\}$, lorsque $d_{\xi}(a, b)$ tend vers l'infini:

$$
\begin{aligned}
& d_{\xi}(a, b)=\exp \left(\frac{1}{2} d\left[\pi_{\xi}(a), \pi_{\xi}(b)\right]+o(1)\right), \\
& \ell_{h}(a, b)=d\left[\pi_{\xi}(a), \pi_{\xi}(b)\right]-2 h+\eta_{h}(a, b),
\end{aligned}
$$

avec $\eta_{h}=\eta_{h}(a, b)$ bornée sur $\left\{\ell_{h}(a, b)>0\right\}$, de limite supérieure $\leq \log 4$, et tendant vers 0 si $\mathrm{e}^{-h} d_{\xi}(a, b)$ tend vers l'infini.

Démonstration. - Fixons $a, b$ distincts dans $\partial X \backslash\{\xi\}$, et deux géodésiques $a_{t}, b_{t}$ partant de $\xi$, telles que $a_{0}=\pi_{\xi}(a), b_{0}=\pi_{\xi}(b) \in H_{0}$, et tendant vers $a, b$ respectivement. Nous avons (voir [12, Appendix]) :

$$
d_{\xi}(a, b)=\lim _{t \rightarrow \infty} \mathrm{e}^{\frac{1}{2}\left(d\left(a_{t}, b_{t}\right)-2 t\right)} .
$$

Nous aurons donc établi la première assertion en montrant que $d\left(a_{0}, b_{0}\right)+$ $2 t-d\left(a_{t}, b_{t}\right) \rightarrow 0$ uniformément par rapport à $t \geq 0$.

Appliquons le lemme 1 au triangle non borné $\left(\xi, a_{t}, b_{t}\right)$ : on obtient $d\left(a_{0}, b_{0}\right) \leq d\left(\bar{a}_{0}, \bar{b}_{0}\right)$, pour $d\left(a_{t}, b_{t}\right)=d\left(\bar{a}_{t}, \bar{b}_{t}\right)$ et $t \geq 0$.

TOME $130-2002-\mathrm{N}^{\mathrm{O}} 3$ 
Comme par inégalité triangulaire $d\left(a_{0}, b_{0}\right)+2 t-d\left(a_{t}, b_{t}\right) \geq 0$, nous n'avons donc plus qu'à majorer $d\left(a_{0}, b_{0}\right)+2 t-d\left(a_{t}, b_{t}\right)$ dans $\mathbb{H}^{2}$. Or dans $\mathbb{H}^{2}$ on trouve

$$
\operatorname{ch}\left[d\left(a_{t}, b_{t}\right)\right]=1+\frac{1}{2} d_{\xi}(a, b)^{2} \mathrm{e}^{2 t},
$$

et donc, uniformément sur $\left\{(a, b, t) \in(\partial X \backslash\{\xi\})^{2} \times \mathbb{R}_{+} \mid d_{\xi}(a, b) \rightarrow \infty\right\}$ :

$$
d\left(a_{t}, b_{t}\right)=2 t+2 \log \left[d_{\xi}(a, b)\right]+o(1)
$$

d'où $d\left(a_{0}, b_{0}\right)+2 t-d\left(a_{t}, b_{t}\right)=o(1)$ (on a même $d\left(a_{0}, b_{0}\right)+2 t-d\left(a_{t}, b_{t}\right)=$ $\mathcal{O}\left(d_{\xi}(a, b)^{-2}\right)$ uniformément sur $\{t \geq 0\}$, dans $X$ comme dans $\left.\mathbb{H}^{2}\right)$, ce qui prouve la première assertion de l'énoncé.

Pour la deuxième moitié de l'énoncé, fixons $h \geq 0$ et $a, b$ avec $d_{\xi}(a, b)$ assez grand pour que la géodésique $] a, b\left[\right.$ rencontre $B_{h}$, successivement en $a_{h}^{\prime}$ et $b_{h}^{\prime}$, de sorte que $\ell_{h}(a, b)=d\left(a_{h}^{\prime}, b_{h}^{\prime}\right)$. Notons $x_{h}$ le point du rayon géodésique $\left[a_{h}^{\prime}, a[\right.$ situé à distance $h$ de $a_{h}^{\prime}$, et de même $y_{h} \in\left[b_{h}^{\prime}, b\left[\right.\right.$ est situé à distance $h$ de $b_{h}^{\prime}$, de sorte que $\ell_{h}(a, b)+2 h=d\left(x_{h}, y_{h}\right)$. Notons enfin $a_{t}$ et $b_{t}$ les intersections de $H_{t}$ avec les géodésiques $] a, \xi[$ et $] b, \xi[$. Nous devons estimer

$$
\eta_{h}(a, b):=\ell_{h}(a, b)+2 h-d\left(\pi_{\xi}(a), \pi_{\xi}(b)\right)=d\left(x_{h}, y_{h}\right)-d\left(a_{0}, b_{0}\right) .
$$

Or

$$
\left|\eta_{h}(a, b)\right| \leq\left|d\left(x_{h}, y_{h}\right)-d\left(x_{h}, b_{0}\right)\right|+\left|d\left(x_{h}, b_{0}\right)-d\left(a_{0}, b_{0}\right)\right| \leq d\left(y_{h}, b_{0}\right)+d\left(x_{h}, a_{0}\right),
$$

et donc en utilisant le lemme 1 dans les triangles $\left(a, a_{h}^{\prime}, a_{h}\right),\left(b, b_{h}^{\prime}, b_{h}\right)$, on obtient $\left|\eta_{h}(a, b)\right| \leq 2 d\left(\bar{x}_{h}, \bar{a}_{0}\right)$, lorsque $\bar{\xi}, \bar{a}, \bar{b}$ sont tels que $d\left(\bar{a}_{h}^{\prime}, \bar{a}_{h}\right)=d\left(a_{h}^{\prime}, a_{h}\right)$. Soient $a_{s}^{\prime \prime} \in\left[a_{h}^{\prime}, a\left[\right.\right.$ et $b_{s}^{\prime \prime} \in\left[a_{h}^{\prime}, b[\right.$ les points de $] a, b\left[\right.$ situés à distance $s$ de $a_{h}^{\prime}$, de sorte que par (1) et (6)

$$
\begin{aligned}
d_{\xi}(a, b) & =\lim _{s, t{ }^{\infty}} \exp \left(t+h+\frac{1}{2}\left[d\left(a_{s}^{\prime \prime}, b_{t}^{\prime \prime}\right)-d\left(a_{s}^{\prime \prime}, a_{t+h}\right)-d\left(b_{t}^{\prime \prime}, a_{t+h}\right)\right]\right) \\
& =\lim _{t \nearrow \infty} \exp \left(t+h+\frac{1}{2}\left[\beta_{a}\left(a_{h}^{\prime}, a_{h}\right)-d\left(b_{t}^{\prime \prime}, a_{t+h}\right)\right]\right) \\
& =\lim _{t \nearrow \infty} \exp \left(t+h+\frac{1}{2}\left[\beta_{\bar{a}}\left(\bar{a}_{h}^{\prime}, \bar{a}_{h}\right)-d\left(b_{t}^{\prime \prime}, a_{t+h}\right)\right]\right) .
\end{aligned}
$$

Or le lemme 1 appliqué de façon contraposée au triangle $\left(a, b_{t}^{\prime \prime}, a_{t+h}\right)$ montre que $d\left(b_{t}^{\prime \prime}, a_{t+h}\right) \geq d\left(\bar{b}_{t}^{\prime \prime}, \bar{a}_{t+h}\right)$, ce qui entraîne que $d_{\xi}(a, b) \leq d_{\bar{\xi}}(\bar{a}, \bar{b})$.

Nous nous sommes donc ramenés à établir dans le cas où $X=\mathbb{H}^{2}$ que $d\left(a_{0}, x_{h}\right)$ est bornée sur $\left\{\ell_{h}(a, b)>0\right\}$ et tend simplement vers 0 lorsque $d_{\xi}(a, b)$ tend vers $\infty$.

Plaçons-nous alors dans le modèle du demi-plan de Poincaré avec $\xi=\infty$ et $H_{0}$ d'ordonnée 1 . Il est clair alors que l'ordonnée de $x_{h}$ est $\geq 1$, et maximale lorsque $] a, b$ [ est tangente à $H_{h}$, auquel cas on calcule aisément qu'elle vaut $2\left(1+\mathrm{e}^{-2 h}\right)^{-1} \leq 2$. De plus nous avons $d_{\xi}(a, b)=|a-b|$. Par conséquent, pour $d_{\xi}(a, b)$ grand, la pente le long de $\left[a_{0}^{\prime}, x_{h}\right]$ est uniformément grande, et donc 
l'écart entre les abscisses de $x_{h}$ et de $a_{0}$ uniformément petit; d'où la borne uniforme sur $\left|a_{0}-x_{h}\right|$ et sur $d\left(a_{0}, x_{h}\right)$, et l'estimation

$$
\limsup _{d_{\xi}(a, b) \rightarrow \infty} d\left(a_{0}, x_{h}\right) \leq \log 2 .
$$

Enfin, pour $h$ fixé, $a_{t}^{\prime}$ converge vers $a_{t}$ uniformément sur $\{0 \leq t \leq h\}$, et donc $d\left(a_{0}, x_{h}\right)$ converge bien vers 0 . En outre un calcul précis montre que $d\left(a_{0}, x_{h}\right) \leq 4|a-b|^{-1}+4 \mathrm{e}^{-2 h}|a-b|^{-2}$.

Considérons l'hypothèse suivante : localement uniformément par rapport à $x, y \in H_{0}$,

$(*) \quad \operatorname{Card}\left\{\gamma \in \Gamma_{\xi} \mid d(x, \gamma y) \leq \varrho\right\} \sim C(x, y) \mathrm{e}^{\delta_{\xi} \varrho} \quad$ lorsque $\varrho \rightarrow+\infty$,

$C$ étant mesurable localement bornée $>0$.

Cette hypothèse sur le groupe $\Gamma_{\xi}$, nécessaire dans la proposition suivante, est automatiquement réalisée lorsque $X$ est un espace symétrique de rang 1 , avec même dans ce cas une fonction $C$ constante. Voir plus loin pour les cas hyperboliques réel et complexe.

Cette hypothèse n'est pas toujours vérifiée, notamment lorsque

$$
\begin{aligned}
\limsup _{\varrho \rightarrow+\infty} \frac{1}{\varrho} \log \operatorname{Card}\left\{\gamma \in \Gamma_{\xi} \mid d(x, \gamma y) \leq \varrho\right\} \\
\quad>\liminf _{\varrho \rightarrow+\infty} \frac{1}{\varrho} \log \operatorname{Card}\left\{\gamma \in \Gamma_{\xi} \mid d(x, \gamma y) \leq \varrho\right\} .
\end{aligned}
$$

T. Roblin discute dans un travail récent [16] l'égalité de ces deux limites, qui a lieu dans le cas général des espaces $\operatorname{CAT}(-1)$, lorsque le groupe n'est pas élémentaire (ce qui n'est pas le cas des groupes paraboliques, et donc de $\Gamma_{\xi}$ ).

Proposition 1. - Considérons pour tous $h>0$ et $r \geq 0$ la série de Poincaré généralisée :

$\Sigma_{h}^{r}:=\int_{\mathcal{D}^{2}} \sum_{\gamma \in \Gamma_{\xi}}\left(1_{] r,+\infty[} \times \phi\right) \circ \ell_{h}\left(v_{-}, \gamma v_{+}\right) \times\left(d_{\xi}\left(v_{-}, \gamma v_{+}\right)\right)^{-2 \delta} \mathrm{d} \mu_{\xi}\left(v_{-}\right) \mathrm{d} \mu_{\xi}\left(v_{+}\right)$,

où $\phi$ est une fonction de classe $C^{2}$ de $\mathbb{R}_{+}$dans $\mathbb{R}$ telle que

$$
\int_{0}^{\infty} \phi_{\varepsilon}^{\prime \prime}(t) \mathrm{e}^{-\left(\delta-\delta_{\xi}\right) t} \mathrm{~d} t<\infty
$$

pour un $\varepsilon>0$, avec $\phi_{\varepsilon}^{\prime \prime}(t):=\sup \left|\phi^{\prime \prime}\right|\left(\left[(t-\varepsilon)^{+}, t+\varepsilon\right]\right)$.

Alors, sous l'hypothèse $\left(^{*}\right)$, nous avons :

$$
\Sigma_{h}^{r}=K_{\mathcal{P}} \delta_{\xi} \int_{r}^{\infty} \phi(t) \mathrm{e}^{-\left(\delta-\delta_{\xi}\right) t} \mathrm{~d} t \times \exp \left(-2\left(\delta-\delta_{\xi}\right) \times(h-\alpha(r, h))\right),
$$

TOME $130-2002-\mathrm{N}^{\mathrm{O}} 3$ 
avec

$$
\begin{gathered}
K_{\mathcal{P}}:=\int_{\mathcal{D}^{2}} C\left(\pi_{\xi}\left(v_{-}\right), \pi_{\xi}\left(v_{+}\right)\right) \mathrm{d} \mu_{\xi}\left(v_{-}\right) \mathrm{d} \mu_{\xi}\left(v_{+}\right), \\
\limsup _{h \rightarrow \infty}|\alpha(r, h)| \leq \log 2, \quad \lim ^{-h_{r \rightarrow \infty}} \alpha(r, h)=0 .
\end{gathered}
$$

Remarque 1. - L'hypothèse sur $\phi$ entraîne que

$$
\begin{aligned}
\int_{0}^{\infty} & |\phi(t)| \mathrm{e}^{-\left(\delta-\delta_{\xi}\right) t} \mathrm{~d} t \\
& \leq|\phi(0)|\left(\delta-\delta_{\xi}\right)^{-1}+\int_{0}^{\infty}\left(\int_{0}^{t}\left|\phi^{\prime}(s)\right| \mathrm{d} s\right) \mathrm{e}^{-\left(\delta-\delta_{\xi}\right) t} \mathrm{~d} t \\
& \leq\left(\delta-\delta_{\xi}\right)^{-1}\left(|\phi(0)|+\int_{0}^{\infty}\left|\phi^{\prime}(t)\right| \mathrm{e}^{-\left(\delta-\delta_{\xi}\right) t} \mathrm{~d} t\right) \\
& \leq\left(\delta-\delta_{\xi}\right)^{-1}\left(|\phi(0)|+\left(\delta-\delta_{\xi}\right)^{-1}\left(\left|\phi^{\prime}(0)\right|+\int_{0}^{\infty}\left|\phi^{\prime \prime}(t)\right| \mathrm{e}^{-\left(\delta-\delta_{\xi}\right) t} \mathrm{~d} t\right)\right)<\infty .
\end{aligned}
$$

Remarque 2. - On ne peut pas remplacer dans le résultat ci-dessus $\alpha(r, h)$ par $o(1)$, comme le montre le résultat de la proposition 6 relative au cas de l'espace hyperbolique réel, pour lequel un calcul plus précis peut être et sera fait.

Remarque 3. - La preuve ci-dessous de la proposition 1 est fondée sur l'hypothèse $(*)$ et le lemme 2 . L'hypothèse $\left(^{*}\right)$ permet donc de donner les expressions asymptotiques recherchées de façon assez explicite. En son absence, la section 4 ci-dessous ne donnerait ces expressions qu'en termes de séries de Poincaré généralisées.

Démonstration. - Observons tout d'abord que sur

$$
\left\{\left(v_{-}, v_{+}, \gamma\right) \in \mathcal{D}^{2} \times \Gamma_{\xi} \mid \ell_{h}\left(v_{-}, \gamma v_{+}\right)>0\right\},
$$

nous avons $h \rightarrow+\infty \Rightarrow d_{\xi}\left(v_{-}, \gamma v_{+}\right) \rightarrow \infty \Leftrightarrow \gamma \rightarrow \infty$.

Nous pouvons donc appliquer le lemme 2, avec $a=v_{-}$et $b=\gamma v_{+}$. Notant en abrégé $d_{\gamma}:=2 \log \left[d_{\xi}\left(v_{-}, \gamma v_{+}\right)\right]$et $\eta_{h}\left(v_{-}, \gamma v_{+}\right)+o(1)=: \eta_{h}$, nous avons ainsi pour tout $\varepsilon>0$ :

$$
\begin{aligned}
\Sigma_{h}^{r} & =\int_{\mathcal{D}^{2}} \sum_{\gamma \in \Gamma_{\xi}}\left(1_{] r,+\infty[} \times \phi\right)\left(d_{\gamma}+\eta_{h}-2 h\right) \mathrm{e}^{-\delta d_{\gamma}} \mathrm{d} \mu_{\xi}\left(v_{-}\right) \mathrm{d} \mu_{\xi}\left(v_{+}\right) \\
& =\int_{\mathcal{D}^{2}} \sum_{\{\varrho \in \varepsilon \mathbb{N} \mid \varrho \geq 2 h+r\}} \sum_{\left\{\gamma \in \Gamma_{\xi} \mid \varrho \leq d_{\gamma}+\eta_{h}<\varrho+\varepsilon\right\}} \phi\left(d_{\gamma}+\eta_{h}-2 h\right) \mathrm{e}^{-\delta d_{\gamma}} \mathrm{d} \mu_{\xi} \otimes \mu_{\xi} \\
& =\int_{\mathcal{D}^{2}} \sum_{\{\varrho \in \varepsilon \mathbb{N} \mid \varrho \geq 2 h+r\}} \sum_{\left\{\gamma \in \Gamma_{\xi} \mid \varrho \leq d_{\gamma}+\eta_{h}<\varrho+\varepsilon\right\}} \phi(\varrho-2 h+\theta \varepsilon) \mathrm{e}^{-\delta\left(\varrho-\eta_{h}+\theta \varepsilon\right)} \mathrm{d} \mu_{\xi} \otimes \mu_{\xi}
\end{aligned}
$$

(pour un certain $\theta=\theta\left(v_{-}, v_{+}, \gamma, h, \varrho, \varepsilon, \phi\right) \in[0,1]$ )

BULletin DE LA SOCiÉtÉ MATHÉmATiQUe DE FRANCE 


$$
\begin{array}{r}
=\int_{\mathcal{D}^{2}} \sum_{\{\varrho \in E \mathbb{N} \mid \varrho \geq 2 h+r\}} \sum_{\left\{\gamma \in \Gamma_{\xi} \mid d_{\gamma}+\eta_{h}<\varrho\right\}}\left[\phi(\varrho-2 h+(\theta-1) \varepsilon) \mathrm{e}^{\delta \varepsilon}\right. \\
-\phi(\varrho-2 h+\theta \varepsilon)] \mathrm{e}^{-\delta\left(\varrho-\eta_{h}+\theta \varepsilon\right)} \\
-\phi(r+\theta \varepsilon) \int_{\mathcal{D}^{2}} \sum_{\left\{\gamma \in \Gamma_{\xi} \mid d_{\gamma}+\eta_{h}<2 h+r\right\}} \mathrm{e}^{-\delta\left(2 h+r-\eta_{h}+\mathcal{O}(\varepsilon)\right)} \mathrm{d} \mu_{\xi} \otimes \mu_{\xi}
\end{array}
$$

(par transformation d'Abel)

$$
\begin{gathered}
=\int_{\mathcal{D}^{2}} \sum_{\{\varrho \in \in \mathbb{N} \mid \varrho \geq 2 h+r\}} \varepsilon\left[\delta \phi(\varrho-2 h)-\phi^{\prime}(\varrho-2 h)+\mathcal{O}(\varepsilon) \phi_{\varepsilon}^{\prime \prime}(\varrho-2 h)\right] \mathrm{e}^{-\delta(\varrho+\mathcal{O}(\varepsilon))} \\
\times \sum_{\left\{\gamma \in \Gamma_{\xi} \mid d_{\gamma}+\eta_{h}<\varrho\right\}} \mathrm{e}^{\delta \eta_{h}} \mathrm{~d} \mu_{\xi} \otimes \mu_{\xi}-\phi(r+\theta \varepsilon) \mathrm{e}^{-\delta(2 h+r)} \\
\times \int_{\mathcal{D}^{2}} \sum_{\left\{\gamma \in \Gamma_{\xi} \mid d_{\gamma}+\eta_{h}<2 h+r\right\}} \mathrm{e}^{\delta \eta_{h}} \mathrm{~d} \mu_{\xi} \otimes \mu_{\xi} \\
=\varepsilon \sum_{\{\varrho \in \varepsilon \mathbb{N} \mid \varrho \geq r\}}\left[\delta \phi(\varrho)-\phi^{\prime}(\varrho)+\mathcal{O}(\varepsilon) \phi_{\varepsilon}^{\prime \prime}(\varrho)\right] \mathrm{e}^{-\delta(\varrho+2 h+\mathcal{O}(\varepsilon))} \\
\times \int_{\mathcal{D}^{2} \sum_{\left\{\gamma \in \Gamma_{\xi} \mid d_{\gamma}+\eta_{h}<\varrho+2 h\right\}} \mathrm{e}^{\delta \eta_{h}} \mathrm{~d} \mu_{\xi} \otimes \mu_{\xi}} \sum_{\mathcal{D}^{2} \sum_{\left\{\gamma \in \Gamma_{\xi} \mid d_{\gamma}+\eta_{h}<2 h+r\right\}} \mathrm{e}^{\delta \eta_{h}} \mathrm{~d} \mu_{\xi} \otimes \mu_{\xi} .}
\end{gathered}
$$

Or l'hypothèse $\left(^{*}\right)$ et le contrôle sur $\eta_{h}$ (venant du lemme 2) assurent que

$\int_{\mathcal{D}^{2}} \sum_{\left\{\gamma \in \Gamma_{\xi} \mid d_{\gamma}+\eta_{h}<\varrho+2 h\right\}} \mathrm{e}^{\delta \eta_{h}} \mathrm{~d} \mu_{\xi} \otimes \mu_{\xi}=K_{\mathcal{P}} \times \exp \left(\delta_{\xi}(\varrho+2 h)+\left(\delta-\delta_{\xi}\right) \alpha(\varrho, h)\right)$,

avec

$$
\limsup _{h \rightarrow \infty}|\alpha(r, h)| \leq \log 4 \quad \text { et } \lim _{\mathrm{e}^{-h} \varrho \rightarrow \infty} \alpha(\varrho, h)=0 .
$$

(En effet, on peut restreindre la somme à $\left\{\gamma \in \Gamma_{\xi} \mid \log (\varrho+h)<d_{\gamma}+\eta_{h}<\right.$ $\varrho+2 h\}$.) Nous obtenons donc pour tout $\varepsilon>0$ :

$$
\begin{aligned}
\Sigma_{h}^{r}= & \varepsilon \sum_{\{\varrho \in \varepsilon \mathbb{N} \mid \varrho \geq r\}}\left[\delta \phi(\varrho)-\phi^{\prime}(\varrho)+\mathcal{O}(\varepsilon) \phi_{\varepsilon}^{\prime \prime}(\varrho)\right] \mathrm{e}^{-\left(\delta-\delta_{\xi}\right)(\varrho+2 h-\alpha(\varrho, h))} K_{\mathcal{P}} \\
= & \sum_{\{\varrho \in \in \mathbb{N} \mid \varrho \geq r\}}\left[\delta \phi(\varrho)-\phi^{\prime}(\varrho)+\mathcal{O}(\varepsilon) \phi_{\varepsilon}^{\prime \prime}(\varrho)\right] \mathrm{e}^{-\left(\delta-\delta_{\xi}\right)(\varrho-\alpha(r, h))} \\
& \left(\operatorname{car}|\phi(r+\theta \varepsilon)-\phi(r)| \mathrm{e}^{-\left(\delta-\delta_{\xi}\right) r} \leq \int_{r}^{r+\varepsilon}\left|\phi^{\prime}(t)\right| \mathrm{e}^{-\left(\delta-\delta_{\xi}\right)(t-\varepsilon)} \mathrm{d} t=\mathcal{O}(\varepsilon)\right)
\end{aligned}
$$

TOME $130-2002-\mathrm{N}^{\mathrm{O}} 3$ 


$$
\begin{aligned}
&=\left(\int_{r}^{\infty}\left[\delta \phi(t)-\phi^{\prime}(t)+\mathcal{O}(\varepsilon) \phi_{\varepsilon}^{\prime \prime}(t)\right] \mathrm{e}^{-\left(\delta-\delta_{\xi}\right) t} \mathrm{~d} t\right.\left.-\phi(r) \mathrm{e}^{-\left(\delta-\delta_{\xi}\right) r}-\mathcal{O}(\varepsilon)\right) \\
& \times K_{\mathcal{P}} \mathrm{e}^{-\left(\delta-\delta_{\xi}\right)(2 h-\alpha(r, h))} \\
&=K_{\mathcal{P}} \mathrm{e}^{-\left(\delta-\delta_{\xi}\right)(2 h-\alpha(r, h))}\left(\delta_{\xi} \int_{r}^{\infty}\left[\phi(t)+\mathcal{O}(\varepsilon) \phi_{\varepsilon}^{\prime \prime}(t)\right] \mathrm{e}^{-\left(\delta-\delta_{\xi}\right) t} \mathrm{~d} t-\mathcal{O}(\varepsilon)\right) \\
&=K_{\mathcal{P}} \mathrm{e}^{-2\left(\delta-\delta_{\xi}\right) h}\left(\delta_{\xi} \int_{r}^{\infty} \phi(t) \mathrm{e}^{-\left(\delta-\delta_{\xi}\right) t} \mathrm{~d} t+\mathcal{O}(\varepsilon)\right) \mathrm{e}^{\left(\delta-\delta_{\xi}\right) \alpha(r, h)}
\end{aligned}
$$

\section{Applications : masse d'une pointe, temps de retour}

4.1. Masse asymptotique de la pointe $\mathcal{P}$. - Rappelons que les mesures de Patterson n'ont pas d'atome au point parabolique $\xi$ d'après l'hypothèse $\delta>\delta_{\xi}$, et qu'elles sont supportées par $\Lambda(\Gamma)$. Il en est donc de même pour la mesure $\mu_{\xi}$. Notons à cette occasion que, de façon générale, dans le cas des groupes géométriquement finis, l'hypothèse de divergence du groupe suffit pour entraîner que $\mu$ n'a pas d'atome (voir dans [5] la proposition 1 et la remarque qui la suit). De sorte que nous avons :

$$
\begin{aligned}
m\left(T^{1} \mathcal{P}_{h}\right) & =\int_{T^{1} B_{h}} 1_{\left\{v_{-} \in \mathcal{D}\right\}} \mathrm{d} \tilde{m}(v) \\
& =\int_{T^{1} B_{h}} 1_{\mathcal{D}}\left(v_{-}\right) d_{\xi}\left(v_{-}, v_{+}\right)^{-2 \delta} \mathrm{d} s \mathrm{~d} \mu_{\xi}\left(v_{-}\right) \mathrm{d} \mu_{\xi}\left(v_{+}\right) \\
& =\int 1_{\mathcal{D}}\left(v_{-}\right) 1_{\Lambda(\Gamma) \backslash\{\xi\}}\left(v_{+}\right) \ell_{h}\left(v_{-}, v_{+}\right) d_{\xi}\left(v_{-}, v_{+}\right)^{-2 \delta} \mathrm{d} \mu_{\xi}\left(v_{-}\right) \mathrm{d} \mu_{\xi}\left(v_{+}\right) \\
& =\sum_{\gamma \in \Gamma_{\xi}} \int 1_{\mathcal{D}}\left(v_{-}\right) 1_{\gamma \mathcal{D}}\left(v_{+}\right) \ell_{h}\left(v_{-}, v_{+}\right) d_{\xi}\left(v_{-}, v_{+}\right)^{-2 \delta} \mathrm{d} \mu_{\xi}\left(v_{-}\right) \mathrm{d} \mu_{\xi}\left(v_{+}\right) \\
& =\sum_{\gamma \in \Gamma_{\xi}} \int 1_{\mathcal{D}}\left(v_{-}\right) 1_{\mathcal{D}}\left(v_{+}\right) \ell_{h}\left(v_{-}, \gamma v_{+}\right) d_{\xi}\left(v_{-}, \gamma v_{+}\right)^{-2 \delta} \mathrm{d} \mu_{\xi}\left(v_{-}\right) \mathrm{d} \mu_{\xi}\left(v_{+}\right)
\end{aligned}
$$

(par $\Gamma_{\xi}$-invariance de $\mu_{\xi}$ )

$$
=\int_{\mathcal{D}^{2}}\left(\sum_{\gamma \in \Gamma_{\xi}} \ell_{h}\left(v_{-}, \gamma v_{+}\right) d_{\xi}\left(v_{-}, \gamma v_{+}\right)^{-2 \delta}\right) \mathrm{d} \mu_{\xi}\left(v_{-}\right) \mathrm{d} \mu_{\xi}\left(v_{+}\right) .
$$

Appliquant la proposition 1 avec $\phi=\operatorname{Id}_{\mathbb{R}_{+}}, r=0$, nous obtenons l'évaluation suivante.

Proposition 2. - Sous l'hypothèse (*), nous avons :

$$
m\left(T^{1} \mathcal{P}_{h}\right)=K_{\mathcal{P}} \delta_{\xi}\left(\delta-\delta_{\xi}\right)^{-2} \mathrm{e}^{-2\left(\delta-\delta_{\xi}\right)\left(h-\alpha_{h}\right)},
$$

avec $\lim \sup _{\{h \rightarrow \infty\}}\left|\alpha_{h}\right| \leq \log 2$. 
4.2. Masse asymptotique des mesures de Palm. - Considérons le système dynamique ergodique $\left(\mathcal{M}, m, \theta_{t}\right)$, où $\theta_{t}$ désigne le flot géodésique sur $T^{1} \mathcal{M}$. Considérons aussi, pour $h>0$, le processus $\left\{\theta_{S_{h}^{2 j+1}} \mid j \in \mathbb{N}\right\}$ des passages successifs du flot géodésique par le sous-fibré transverse $T_{+}^{1} \mathcal{P}_{h}$ constitué des éléments de $T^{1} \mathcal{M}$ basés sur $\partial \mathcal{P}_{h}$ et pointant vers $\mathcal{P}_{h}$.

Notons $\chi_{h}$ la mesure de Palm associée à ce processus (voir par exemple [6]) ; on peut la définir par désintégration par rapport au temps de la mesure $m$, dans des coordonnées exprimant le flot géodésique comme suspension de l'application de premier retour dans $T_{+}^{1} \mathcal{P}_{h}$. D'après le théorème d'Ambrose (voir [6, exposés I et II]), $\chi_{h}$ est invariante pour le processus $\left\{\theta_{S_{h}^{2 j+1}} \mid j \in \mathbb{N}\right\}$, et le système dynamique $\left(T_{+}^{1} \mathcal{P}_{h}, \chi_{h}, \theta_{S_{h}^{2 j+1}}\right)$ est ergodique.

Nous voulons obtenir un équivalent asymptotique (lorsque $h \rightarrow \infty$ ) de la masse $\left|\chi_{h}\right|$ de la mesure de Palm $\chi_{h}$.

Or l'ensemble des entrées dans $\mathcal{P}_{h}$ des géodésiques de $\mathcal{M}$ s'identifie successivement avec l'ensemble des entrées dans tous les domaines fondamentaux de $\mathcal{P}_{h}$ des classes modulo $\Gamma$ de géodésiques de $X$; avec l'ensemble des entrées dans un domaine fondamental fixé de $\mathcal{P}_{h}$ des géodésiques de $X$; avec l'ensemble des entrées dans les images par $\Gamma_{\xi}$ d'un domaine fondamental fixé de $\mathcal{P}_{h}$ des classes modulo $\Gamma_{\xi}$ de géodésiques de $X$; avec l'ensemble des entrées dans $B_{h}$ des géodésiques de $X$ issues d'un domaine fondamental de $\Gamma_{\xi} \backslash \partial X$. De sorte que nous avons par définition de $\chi_{h}$ :

$$
\begin{aligned}
\left|\chi_{h}\right| & =\int 1_{\mathcal{D}}\left(v_{-}\right) 1_{\Lambda(\Gamma) \backslash\{\xi\}}\left(v_{+}\right) 1_{\left\{\ell_{h}\left(v_{-}, v_{+}\right)>0\right\}} d_{\xi}\left(v_{-}, v_{+}\right)^{-2 \delta} \mathrm{d} \mu_{\xi}\left(v_{-}\right) \mathrm{d} \mu_{\xi}\left(v_{+}\right) \\
& =\sum_{\gamma \in \Gamma_{\xi}} \int 1_{\mathcal{D}}\left(v_{-}\right) 1_{\gamma \mathcal{D}}\left(v_{+}\right) 1_{\left\{\ell_{h}\left(v_{-}, v_{+}\right)>0\right\}} d_{\xi}\left(v_{-}, v_{+}\right)^{-2 \delta} \mathrm{d} \mu_{\xi}\left(v_{-}\right) \mathrm{d} \mu_{\xi}\left(v_{+}\right) \\
& =\sum_{\gamma \in \Gamma_{\xi}} \int 1_{\mathcal{D}}\left(v_{-}\right) 1_{\mathcal{D}}\left(v_{+}\right) 1_{\left\{\ell_{h}\left(v_{-}, \gamma v_{+}\right)>0\right\}} d_{\xi}\left(v_{-}, \gamma v_{+}\right)^{-2 \delta} \mathrm{d} \mu_{\xi}\left(v_{-}\right) \mathrm{d} \mu_{\xi}\left(v_{+}\right) \\
& =\int_{\mathcal{D}^{2}}\left(\sum_{\gamma \in \Gamma_{\xi}} 1_{\mathbb{R}_{+}^{*}} \circ \ell_{h}\left(v_{-}, \gamma v_{+}\right) \times d_{\xi}\left(v_{-}, \gamma v_{+}\right)^{-2 \delta}\right) \mathrm{d} \mu_{\xi}\left(v_{-}\right) \mathrm{d} \mu_{\xi}\left(v_{+}\right) .
\end{aligned}
$$

Appliquant la proposition 1 avec $\phi \equiv 1, r=0$, nous obtenons l'évaluation suivante.

Proposition 3. - Sous l'hypothèse $\left({ }^{*}\right)$, nous avons :

$$
\left|\chi_{h}\right|=K_{\mathcal{P}} \delta_{\xi}\left(\delta-\delta_{\xi}\right)^{-1} \mathrm{e}^{-2\left(\delta-\delta_{\xi}\right)\left(h-\alpha_{h}\right)},
$$

avec $\lim \sup _{\{h \rightarrow \infty\}}\left|\alpha_{h}\right| \leq \log 2$.

Remarque. - $\left|\chi_{h}\right| /|m|$ est également la probabilité (sous $m /|m|$ ) que la pénétration maximale $\eta$ de l'excursion générique dans $\mathcal{P}_{0}$ dépasse $h$. Précisément,

TOME $130-2002-\mathrm{N}^{\mathrm{O}} 3$ 
si $e=\left\{e_{t} \mid t \in\left[S, S^{\prime}\right]\right\}$ est une excursion générique du flot géodésique dans $\mathcal{P}_{0}$,

$$
\eta(e):=\max _{t} d\left(\partial \mathcal{P}_{0}, e_{t}\right)
$$

vérifie $\int_{T^{1} \mathcal{M}} 1_{\{\eta \geq h\}} \mathrm{d} m=\left|\chi_{h}\right|$ pour tout $h>0$. Donc la proposition 3 donne une estimation de la queue de la loi de $\eta$.

4.3. Asymptotique des temps de retour près de $\mathcal{P}$. - Nous avons introduit dans la section précédente la suite $\left\{S_{h}^{2 j+1} \mid j \in \mathbb{N}\right\}$ des temps d'entrée du flot géodésique dans $\mathcal{P}_{h}$. Notons de plus $S_{h}^{2 j}$ le $j$-ème temps de sortie de $\mathcal{P}_{h}$, de sorte que $0 \leq S_{h}^{2 j}<S_{h}^{2 j+1}<S_{h}^{2 j+2}$ pour tout $j \in \mathbb{N}$ et tout $h>0$.

Appliquant deux fois le théorème ergodique, nous avons pour chaque $h>0$

$$
\begin{aligned}
& \lim _{N \rightarrow \infty}\left(S_{h}^{2 N}\right)^{-1} \sum_{j=1}^{N}\left(S_{h}^{2 j}-S_{h}^{2 j-1}\right)=|m|^{-1} \times m\left(T^{1} \mathcal{P}_{h}\right) m-p . s . \\
& \lim _{N \rightarrow \infty} N^{-1} \sum_{j=1}^{N}\left(S_{h}^{2 j}-S_{h}^{2 j-1}\right)=\left|\chi_{h}\right|^{-1} \times \int \tau \mathrm{d} \chi_{h} \chi_{h}-p . s .
\end{aligned}
$$

où $\tau$ désigne le temps de retour sur l'horocycle $\partial \mathcal{P}_{h}$.

Or nous avons par définition de $\tau$ et de $\chi_{h}$ :

$$
\int \tau \mathrm{d} \chi_{h}=\iint_{0}^{\tau} \mathrm{d} s \mathrm{~d} \chi_{h}=m\left(T^{1} \mathcal{P}_{h}\right)
$$

Nous déduisons donc que pour chaque $h>0$

$$
\lim _{N \rightarrow \infty} \frac{S_{h}^{2 N-1}}{N}=\lim _{N \rightarrow \infty} \frac{S_{h}^{2 N}}{N}=\frac{|m|}{\left|\chi_{h}\right|} \cdot m-p . s .
$$

$\mathrm{Au}$ vu de la proposition 3, nous avons établi l'asymptotique suivant des temps de retour près de $\mathcal{P}$.

Proposition 4. - Sous l'hypothèse $\left({ }^{*}\right)$, nous avons m-presque sûrement :

$$
\lim _{N \rightarrow \infty} \frac{S_{h}^{2 N-1}}{N}=\lim _{N \rightarrow \infty} \frac{S_{h}^{2 N}}{N}=|m| K_{\mathcal{P}}^{-1} \delta_{\xi}^{-1}\left(\delta-\delta_{\xi}\right) \mathrm{e}^{2\left(\delta-\delta_{\xi}\right)\left(h-\alpha_{h}\right)},
$$

avec $\lim \sup _{\{h \rightarrow \infty\}}\left|\alpha_{h}\right| \leq \log 2$.

De ce qui précède, on déduit aussi l'asymptotique du temps passé dans $\mathcal{P}_{h}$ sous la probabilité $m /|m|$ : cela vaut

$$
\frac{m\left(T^{1} \mathcal{P}_{h}\right)}{\left|\chi_{h}\right|}=\left(\delta-\delta_{\xi}\right)^{-1} \mathrm{e}^{4\left(\delta-\delta_{\xi}\right) \alpha_{h}} .
$$

Nous pouvons en fait, par la même méthode que précédemment, évaluer directement la loi asymptotique de la durée d'une excursion du flot géodésique dans $\mathcal{P}_{h}$.

BULLETIN DE LA SOCIÉtÉ MATHÉMATIQUE DE FRANCE 
Proposition 5. - Sous l'hypothèse $\left(^{*}\right)$ et sous la probabilité $m /|m|$, la queue de la loi de la durée $S_{h}^{2 j}-S_{h}^{2 j-1}$ d'une excursion du flot géodésique dans $\mathcal{P}_{h}$ est (pour tout $h>0$ ) exponentielle de paramètre $\delta-\delta_{\xi}$.

Démonstration. — Procédant comme à la section 4.2, nous avons pour tout réel $r \geq 0$ :

$$
\begin{aligned}
|m|^{-1} \int 1_{\left\{S_{h}^{2 j}-S_{h}^{2 j-1}>r\right\}} \mathrm{d} m=\left|\chi_{h}\right|^{-1} \int 1_{\{\tau>r\}} \mathrm{d} \chi_{h} \\
\quad=\left|\chi_{h}\right|^{-1} \int_{\mathcal{D} \times(\Lambda(\Gamma) \backslash\{\xi\})} 1_{\left\{\ell_{h}\left(v_{-}, v_{+}\right)>r\right\}} \mathrm{d}_{\xi}\left(v_{-}, v_{+}\right)^{-2 \delta} \mathrm{d} \mu_{\xi}\left(v_{-}\right) \mathrm{d} \mu_{\xi}\left(v_{+}\right) \\
=\left|\chi_{h}\right|^{-1} \int_{\mathcal{D}^{2}} \sum_{\gamma \in \Gamma_{\xi}} 1_{\left\{\ell_{h}\left(v_{-}, \gamma v_{+}\right)>r\right\}} d_{\xi}\left(v_{-}, \gamma v_{+}\right)^{-2 \delta} \mathrm{d} \mu_{\xi}\left(v_{-}\right) \mathrm{d} \mu_{\xi}\left(v_{+}\right) \\
\sim\left|\chi_{h}\right|^{-1} K_{\mathcal{P}} \delta_{\xi}\left(\delta-\delta_{\xi}\right)^{-1} \mathrm{e}^{-\left(\delta-\delta_{\xi}\right) r} \mathrm{e}^{-2\left(\delta-\delta_{\xi}\right) h} \sim \mathrm{e}^{-\left(\delta-\delta_{\xi}\right)\left(r-2 \alpha_{h}\right)}
\end{aligned}
$$

lorsque $r \rightarrow+\infty\left(\operatorname{avec} \lim \sup _{h}\left|\alpha_{h}\right| \leq \log 2\right)$, par les propositions 1 et 3 .

\section{Cas hyperbolique réel de la courbure constante}

Nous considérons ici le cas où $X=\mathbb{H}$ est l'espace hyperbolique réel de dimension $d+1$, et donc est de courbure $\kappa \equiv-1$.

Il est bien connu que $\Gamma_{\xi}$ admet un sous-groupe abélien maximal $\Gamma_{\xi}^{\prime}$ d'indice fini $n$, isomorphe à $\mathbb{Z}^{k}$. En particulier nous avons $\delta_{\xi}=\frac{1}{2} k$.

Utilisant le modèle du demi-espace de Poincaré avec $\xi=\infty$ et $H_{0}$ à ordonnée 1 , et identifiant les translations de $\Gamma_{\xi}^{\prime}$ à leur vecteur, nous avons pour $x, y \in H_{0}$ variant dans un compact et $\gamma \in \Gamma_{\xi}^{\prime}$ grand

$$
d(x, \gamma y)=\operatorname{Argch}\left(1+\frac{1}{2}|x-y-\gamma|^{2}\right)
$$

et donc pour $\varrho$ grand

$$
\begin{aligned}
\operatorname{Card}\left\{\gamma \in \Gamma_{\xi}^{\prime} \mid d(x, \gamma y) \leq \varrho\right\} & =\operatorname{Card}\left\{\gamma \in \Gamma_{\xi}^{\prime} \mid(|\gamma|+\mathcal{O}(1))^{2} \leq 2 \operatorname{ch} \varrho\right\} \\
& =\left((2 \operatorname{ch} \varrho)^{1 / 2}-\mathcal{O}(1)\right)^{k} \frac{\left|B^{k}\right|}{\left|\Gamma_{\xi}^{\prime} \backslash \mathbb{R}^{k}\right|} \\
& =\frac{\left|B^{k}\right|}{\left|\Gamma_{\xi}^{\prime} \backslash \mathbb{R}^{k}\right|} \mathrm{e}^{\frac{1}{2} \varrho k}+\mathcal{O}\left(\mathrm{e}^{\frac{1}{2} \varrho(k-1)}\right),
\end{aligned}
$$

où $\left|B^{k}\right|=\pi^{\frac{1}{2} k} / \Gamma\left(1+\frac{1}{2} k\right)$ est le volume de la boule unité de $\mathbb{R}^{k}$ et $\left|\Gamma_{\xi}^{\prime} \backslash \mathbb{R}^{k}\right|$ est le covolume euclidien de $\Gamma_{\xi}^{\prime}$.

TOME $130-2002-\mathrm{N}^{\mathrm{O}} 3$ 
Notant $\gamma_{1}, \ldots, \gamma_{n}$ des représentants de $\Gamma_{\xi} / \Gamma_{\xi}^{\prime}$, nous déduisons que pour $x, y$ variant dans un compact de $H_{0}$ et $\varrho$ grand :

$$
\begin{aligned}
\operatorname{Card}\left\{\gamma \in \Gamma_{\xi} \mid d(x, \gamma y) \leq \varrho\right\} & =\sum_{j=1}^{n} \operatorname{Card}\left\{\gamma \in \Gamma_{\xi}^{\prime} \mid d\left(x, \gamma \gamma_{j} y\right) \leq \varrho\right\} \\
& =\frac{\left(\pi \mathrm{e}^{\varrho}\right)^{\frac{1}{2} k}+\mathcal{O}\left(\mathrm{e}^{\frac{1}{2} \varrho(k-1)}\right)}{\Gamma\left(1+\frac{1}{2} k\right) \cdot\left|\Gamma_{\xi} \backslash \mathbb{R}^{k}\right|},
\end{aligned}
$$

$\left|\Gamma_{\xi} \backslash \mathbb{R}^{k}\right|=\left|\Gamma_{\xi}^{\prime} \backslash \mathbb{R}^{k}\right| / n=\operatorname{Covol}\left(\Gamma_{\xi}\right)$ étant le covolume euclidien de $\Gamma_{\xi}$.

Ceci montre que l'hypothèse $(*)$ est bien vérifiée dans le cas présent, avec

$$
C(x, y) \equiv C_{\mathcal{P}}:=\frac{\pi^{\frac{1}{2} k}}{\Gamma\left(1+\frac{1}{2} k\right) \operatorname{Covol}\left(\Gamma_{\xi}\right)}
$$

qui ne dépend que de la pointe $\mathcal{P}$.

Toujours dans le cadre du modèle de Poincaré précisé ci-dessus, nous avons pour $v_{-}, v_{+} \in \partial \mathbb{H} \backslash\{\infty\}$, avec les notations de la preuve de la proposition 1 :

$$
\ell_{h}\left(v_{-}, \gamma v_{+}\right)=2 \operatorname{Argch}\left(\frac{\left|v_{-}-\gamma v_{+}\right|}{2 \mathrm{e}^{h}}\right)=2 \operatorname{Argch}\left(\frac{1}{2} \mathrm{e}^{\frac{1}{2}\left(d_{\gamma}-2 h\right)}\right) ;
$$

donc, dans l'expression de la série $\Sigma_{h}^{r}$ au début de la preuve de la proposition 1, nous pouvons remplacer $\eta_{h}$ par 0 , à condition de remplacer $\phi$ par $\phi \circ\left(2 \operatorname{Argch}\left(\frac{1}{2} \mathrm{e}^{\frac{1}{2} \mathrm{Id}}\right)\right)$ et $r$ par $2 \log \left(2 \operatorname{ch}\left(\frac{1}{2} r\right)\right)$.

5.1. Masse des pointes et temps de retour. - Nous déduisons de ce qui précède le résultat suivant, qui précise la proposition 1 dans le cas de la courbure constante :

Proposition 6. - Considérons pour tous $h>0$ et $r \geq 0$ la série de Poincaré généralisée

$$
\Sigma_{h}^{r}:=\int_{\mathcal{D}^{2}} \sum_{\gamma \in \Gamma_{\xi}}\left(1_{1 r,+\infty[} \times \phi\right) \circ \ell_{h}\left(v_{-}, \gamma v_{+}\right)\left(d_{\xi}\left(v_{-}, \gamma v_{+}\right)\right)^{-2 \delta} \mathrm{d} \mu_{\xi}\left(v_{-}\right) \mathrm{d} \mu_{\xi}\left(v_{+}\right),
$$

où $\phi$ est une fonction de classe $C^{2}$ de $\mathbb{R}_{+}$dans $\mathbb{R}$ telle que

$$
\int_{0}^{\infty} \phi_{\varepsilon}^{\prime \prime}(t) \mathrm{e}^{-\left(\delta-\delta_{\xi}\right) t} \mathrm{~d} t<\infty
$$

pour un $\varepsilon>0$, avec $\phi_{\varepsilon}^{\prime \prime}(t):=\sup \left|\phi^{\prime \prime}\right|\left(\left[(t-\varepsilon)^{+}, t+\varepsilon\right]\right)$.

Alors nous avons lorsque $h \rightarrow \infty$ :

$\Sigma_{h}^{r}=C_{\mathcal{P}} \lambda_{\mathcal{P}}^{2} \frac{k}{2} \int_{r}^{\infty} \phi(u)\left(2 \operatorname{ch}\left(\frac{1}{2} u\right)\right)^{k-2 \delta} \operatorname{th}\left(\frac{1}{2} u\right) \mathrm{d} u \times \exp ((k-2 \delta) h)\left(1+\mathcal{O}\left(\mathrm{e}^{-h}\right)\right)$.

Note. - Rappellons que la quantité $\lambda_{\mathcal{P}}$, cruciale dans toutes les estimations suivantes, a été introduite au paragraphe 3, et désigne la masse de la mesure induite par $\mu_{\xi}$ sur $\Gamma_{\xi} \backslash \Lambda(\Gamma)$. 
REMARQUE. - Cette estimation précise est fondée sur une relation exacte entre $\ell_{h}$ et $d_{\gamma}$, et n'a donc pas exactement la même forme que dans l'estimation de la proposition 1. En particulier la fonction contre laquelle $\phi$ est intégrée dans le résultat est différente.

De même qu'à la section 4, la masse asymptotique de la pointe se déduit de là en choisissant $r=0$ et $\phi=\mathrm{Id}$. Intégrant par parties et posant $v:=1-$ th $u$, on obtient :

$$
\begin{gathered}
\int_{0}^{\infty} u\left(\operatorname{ch}\left(\frac{1}{2} u\right)\right)^{k-2 \delta} \operatorname{th}\left(\frac{1}{2} u\right) \mathrm{d} u=4 \int_{0}^{\infty} u(\operatorname{ch} u)^{k-2 \delta-1} \operatorname{sh} u \mathrm{~d} u \\
=\frac{4}{2 \delta-k} \int_{0}^{\infty}(\operatorname{ch} u)^{k-2 \delta} \mathrm{d} u=\frac{4}{2 \delta-k} \int_{0}^{1}(v(2-v))^{\delta-1-\frac{1}{2} k} \mathrm{~d} v \\
=\frac{2^{2 \delta-k}}{2 \delta-k} \int_{0}^{1}(v(1-v))^{\delta-1-\frac{1}{2} k} \mathrm{~d} v=\frac{2^{2 \delta-k} \Gamma\left(\delta-\frac{1}{2} k\right)^{2}}{\Gamma(2 \delta-k+1)}
\end{gathered}
$$

Nous avons ainsi la version précisée suivante de la proposition 2 :

Corollaire 1. - Nous avons lorsque $h \rightarrow \infty:$

$$
m\left(T^{1} \mathcal{P}_{h}\right)=C_{\mathcal{P}} \lambda_{\mathcal{P}}^{2} \frac{k \Gamma\left(\delta-\frac{1}{2} k\right)^{2}}{2 \Gamma(2 \delta-k+1)} \mathrm{e}^{(k-2 \delta) h}\left(1+\mathcal{O}\left(\mathrm{e}^{-h}\right)\right) .
$$

De même qu'à la section 4, la masse asymptotique de la mesure de Palm se déduit en choisissant $r=0$ et $\phi \equiv 1$. Nous avons ainsi la version précisée de la proposition 3, donnant la masse de $\left|\chi_{h}\right|$ et par là-même (voir la remarque de la section 4.2) la queue de la loi sous $m /|m|$ de la pénétration maximale $\eta$ de l'excursion générique dans $\mathcal{P}_{0}$ :

Corollaire 2. - Nous avons lorsque $h \rightarrow \infty:$

$$
\left|\chi_{h}\right|=C_{\mathcal{P}} \lambda_{\mathcal{P}}^{2} \frac{2^{k-2 \delta} k}{2 \delta-k} \mathrm{e}^{(k-2 \delta) h}\left(1+\mathcal{O}\left(\mathrm{e}^{-h}\right)\right)
$$

De même qu'à la section 4 , on en déduit la version précisée de la proposition 4 :

Corollaire 3. - Nous avons m-presque sûrement:

$$
\lim _{N \rightarrow \infty} \frac{S_{h}^{2 N-1}}{N}=\lim _{N \rightarrow \infty} \frac{S_{h}^{2 N}}{N}=|m| C_{\mathcal{P}}^{-1} \lambda_{\mathcal{P}}^{-2} \frac{2 \delta-k}{2^{k-2 \delta} k} \mathrm{e}^{(2 \delta-k) h} \times\left(1+\mathcal{O}\left(\mathrm{e}^{-h}\right)\right) .
$$

Nous avons ensuite la version précisée de la proposition 5 :

Corollaire 4. - Sous la probabilité $m /|m|$, la loi de la durée $\left(S_{h}^{2 j}-S_{h}^{2 j-1}\right)$ d'une excursion du flot géodésique dans $\mathcal{P}_{h}$ converge lorsque $h \rightarrow \infty$ vers la loi de densité

$$
t \longmapsto\left(\delta-\frac{1}{2} k\right)\left(\operatorname{ch} \frac{1}{2} t\right)^{k-2 \delta-1} \operatorname{sh}\left(\frac{1}{2} t\right) .
$$

TOME $130-2002-\mathrm{N}^{\mathrm{O}} 3$ 
Démonstration. - Procédant comme aux sections 5 et 6 , et appliquant la proposition 6 avec $r=0$ et $\phi(u)=\mathrm{e}^{\sqrt{-1} q u}$, puis le corollaire 2 , nous avons pour tout réel $q$ :

$$
\begin{gathered}
|m|^{-1} \int \exp \left(\sqrt{-1} q\left(S_{h}^{2 j}-S_{h}^{2 j-1}\right)\right) \mathrm{d} m=\left|\chi_{h}\right|^{-1} \int \exp (\sqrt{-1} q \tau) \mathrm{d} \chi_{h} \\
=\left(\delta-\frac{1}{2} k\right) \int_{0}^{\infty} \mathrm{e}^{\sqrt{-1} q t}\left(\operatorname{ch} \frac{1}{2} t\right)^{k-2 \delta-1} \operatorname{sh}\left(\frac{1}{2} t\right) \mathrm{d} t+\mathcal{O}\left(\mathrm{e}^{-h}\right) .
\end{gathered}
$$

5.2. Loi asymptotique de l'enroulement. - Fixons une base $v_{1}, \ldots, v_{k}$ du réseau $\Gamma_{\infty}^{\prime} \equiv \mathbb{Z}^{k} \subset \mathbb{R}^{k}$, et écrivons $\mathbb{R}^{d}=\mathbb{R}^{k} \oplus \mathbb{R}^{d-k}$. Considérons $\omega_{1}, \ldots, \omega_{k}$ les formes harmoniques constituant la base duale de $v_{1}, \ldots, v_{k}$.

Nous appellerons enroulement associé à une forme

$$
\omega:=\sum_{j=1}^{k} \theta^{j} \omega_{j}
$$

pour $\theta \in \mathbb{R}^{k}$, l'intégrale de $\omega$ le long d'une excursion générique du flot géodésique dans $\mathcal{P}_{h}$; cela peut s'écrire $\int_{g} 1_{\left[S_{h}^{3}, S_{h}^{4}\right]} \omega$, où $g$ désigne une géodésique générique de $\mathcal{M}$.

Nous appellerons demi-enroulement la moitié de cette quantité.

Nota Bene. - Les formes $\omega_{1}, \ldots, \omega_{k}$ ne constituent pas une base de la cohomologie de $\mathcal{P}_{h}$, bien que ceci soit vrai en dimension 2 ou 3 , car $\Gamma_{\xi}$ est alors abélien, ce qui rend le groupe d'homologie de la pointe isomorphe à $\Gamma_{\xi}$. Par contre, en dimension 4 , le groupe d'isométries de $\mathbb{R}^{3} \equiv \partial \mathbb{H}^{4}$ engendré par la symétrie-translation par rapport à $\mathbb{R} e_{1}$ et de vecteur $e_{1}$ et par la translation de vecteur $e_{2}$ a son sous-groupe dérivé engendré par la translation de vecteur $2 e_{2}$; le quotient $\Gamma_{\xi} /\left[\Gamma_{\xi}, \Gamma_{\xi}\right]$ est alors le produit de $\mathbb{Z}$ par un groupe fini; donc dans cet exemple le rang géométrique est 2 , tandis que le premier nombre de Betti du groupe est 1 .

Désignant par $] v_{-}, v_{+}$[ la géodésique de $\mathbb{H}$ relevant $g$ et par $x_{1}, \ldots, x_{k}$ les coordonnées dans la base $v_{1}, \ldots, v_{k}$ de $\mathbb{R}^{k}$, nous avons

$$
\int_{g} 1_{\left[S_{h}^{3}, S_{h}^{4}\right]} \omega=\int_{v_{-}}^{v_{+}} 1_{B_{h}} \sum_{j=1}^{k} \theta^{j} \mathrm{~d} x_{j}=2 \sum_{j=1}^{k} \theta^{j} Y_{h}^{j}\left(v_{-}, v_{+}\right),
$$

où $Y_{h}^{j}\left(v_{-}, v_{+}\right)$est la $j$-ième coordonnée dans la base $v_{1}, \ldots, v_{k}$ de la projection sur $\mathbb{R}^{k}$ parallèlement à $\mathbb{R}^{d-k}$ du vecteur $Y_{h}\left(v_{-}, v_{+}\right) \in \mathbb{R}^{d}$ représentant le demi-enroulement produit dans l'horoboule $B_{h}$ par la géodésique ] $v_{-}, v_{+}$[ d'extrémités $v_{-}, v_{+}$.

BULletin DE LA SOCIÉtÉ MATHÉMATIQUE DE FRANCE 
Nous voyons donc que l'enroulement d'une excursion dans $\mathcal{P}_{h}$ du flot géodésique sur $\mathcal{M}$ est totalement décrite par la variable $Y_{h}$.

Utilisant toujours le modèle du demi-espace de Poincaré avec $\xi=\infty$ et $H_{0}$ à ordonnée 1 , nous avons

$$
\begin{aligned}
Y_{h}\left(v_{-}, v_{+}\right) & =\left(\left|\frac{v_{+}-v_{-}}{2}\right|^{2}-\mathrm{e}^{2 h}\right)^{1 / 2} \cdot \frac{v_{+}-v_{-}}{\left|v_{+}-v_{-}\right|} \\
& =\mathrm{e}^{h} \operatorname{sh}\left(\frac{\ell_{h}\left(v_{-}, v_{+}\right)}{2}\right) \cdot \frac{v_{+}-v_{-}}{\left|v_{+}-v_{-}\right|} \in \mathbb{R}^{d}
\end{aligned}
$$

Rappelons en effet que $\mathrm{e}^{h}$ est la hauteur euclidienne de l'horocycle $H_{h}=\partial B_{h}$, et que $\ell_{h}\left(v_{-}, v_{+}\right)=2 \operatorname{Argch}\left(\left|v_{+}-v_{-}\right| / 2 \mathrm{e}^{h}\right)$.

Le théorème 1 ci-dessous donne la loi asymptotique de $\mathrm{e}^{-h} Y_{h}$, et donc décrit exactement la loi asymptotique sous $m$ de l'enroulement d'une excursion dans $\mathcal{P}_{h}$ du flot géodésique sur $\mathcal{M}$.

ThÉORÈme 1. - Sous la probabilité invariante $m /|m|$, la loi du demienroulement normalisé $\mathrm{e}^{-h} Y_{h}$ de la première excursion complète accomplie par le flot géodésique dans $\mathcal{P}_{h}$ converge lorsque $h \rightarrow+\infty$ vers la loi isotrope sur $\mathbb{R}^{k}$ qui a pour densité

$$
w \longmapsto \pi^{-\frac{1}{2} k} \Gamma\left(\frac{1}{2} k\right)\left(\delta-\frac{1}{2} k\right)\left(1+|w|^{2}\right)^{\frac{1}{2} k-\delta-1}|w|^{2-k} .
$$

Remarques. - 1) Cette loi asymptotique des enroulements près de $\mathcal{P}$, qui se trouve dans le domaine d'attraction (voir [8]) d'une loi stable isotrope d'exposant $\min \{2,(2 \delta-k)\}$ n'est pas elle-même stable.

2) Nous avons entre l'enroulement global $Y_{h}$ et la durée $\tau_{h}$ d'une excursion dans $\mathcal{P}_{h}$ la relation algébrique déterministe $\tau_{h}=2 \operatorname{Argsh}\left(\frac{1}{2}\left|Y_{h}\right|\right)$, qui permet de déduire du théorème 1 le corollaire 4 , et aussi de conclure que ces deux convergences en loi, celle du théorème 1 et celle du corollaire 4 , ont lieu conjointement.

3) On vérifie bien qu'il n'y a pas d'enroulement asymptotique dans la direction de $\mathbb{R}^{d-k}$.

Démonstration. - Dans l'orbifold $\mathcal{M}$, il revient au même de considérer la loi de l'enroulement produit sous la probabilité $m /|m|$ par la première excursion complète dans $\mathcal{P}_{h}$, ou la loi de l'enroulement produit sous la probabilité $\chi_{h} /\left|\chi_{h}\right|$ (voir la section 4.2).

TOME $130-2002-\mathrm{N}^{\mathrm{O}} 3$ 
Nous cherchons donc, pour tout $\theta \in \mathbb{R}^{d}$ fixé, la limite lorsque $h \rightarrow \infty$ de

$$
\begin{aligned}
& Z_{h}^{\theta}:=\int \exp \left(\sqrt{-1} \theta \mathrm{e}^{-h} Y_{h}\right) \frac{\mathrm{d} m}{|m|}=\int \exp \left(\sqrt{-1} \theta \mathrm{e}^{-h} Y_{h}\right) \frac{\mathrm{d} \chi_{h}}{\left|\chi_{h}\right|} \\
&=\frac{1}{\left|\chi_{h}\right|} \int_{\mathcal{D} \times \Lambda(\Gamma)} 1_{\left\{\ell_{h}\left(v_{-}, v_{+}\right)>0\right\}} \exp \left(\sqrt{-1} \theta \mathrm{e}^{-h} Y_{h}\left(v_{-}, v_{+}\right)\right) \\
&=\frac{1}{\left|\chi_{h}\right|} \int_{\mathcal{D}^{2}} \sum_{\gamma \in \Gamma_{\infty}} 1_{\left\{\ell_{h}\left(v_{-}, \gamma v_{+}\right)>0\right\}} \exp \left(\sqrt{-1} \frac{\theta Y_{h}\left(v_{-}, \gamma v_{+}\right)}{\mathrm{e}^{h}}\right) \\
& \mathrm{d}_{\infty}\left(v_{-}, \gamma v_{+}\right)^{-2 \delta} \mathrm{d} \mu_{\infty} \otimes \mathrm{d} \mu_{\xi} \\
& \mathrm{d} \mu_{\infty}
\end{aligned}
$$

Considérons d'abord la seule somme :

$$
\begin{aligned}
\Sigma_{h}^{\prime} & =\Sigma_{h}^{\prime}\left(v_{-}, v_{+}, \theta\right) \\
& :=\sum_{\gamma \in \Gamma_{\infty}^{\prime}} 1_{\left\{\ell_{h}\left(v_{-}, \gamma v_{+}\right)>0\right\}} \exp \left(\sqrt{-1} \theta Y_{h}\left(v_{-}, \gamma v_{+}\right)\right) \mathrm{d}_{\infty}\left(v_{-}, \gamma v_{+}\right)^{-2 \delta}
\end{aligned}
$$

où $v_{-}, v_{+}$varient dans le compact $\overline{\mathcal{D}}, \theta \in \mathbb{R}^{d}$, et $h \rightarrow+\infty$.

Du fait du terme angulaire $\left(v_{+}-v_{-}\right) /\left|v_{+}-v_{-}\right|$présent dans $Y_{h}, \Sigma_{h}^{\prime}$ n'est pas une série de Poincaré généralisée au sens des sections précédentes. En conséquence de quoi, nous allons procéder un peu différemment pour traiter $\Sigma_{h}^{\prime}$, préférant cette fois la méthode des rectangles.

Notons $v_{0}:=v_{+}-v_{-}$, qui varie dans un compact fixe. Nous avons

$$
\begin{aligned}
\Sigma_{h}^{\prime}= & \sum_{\ell \in \mathbb{Z}^{k}} 1_{\left\{\left|v_{0}+\sum_{j=1}^{k} \ell_{j} v_{j}\right|>2 \mathrm{e}^{h}\right\}} \cdot\left|v_{0}+\sum_{j=1}^{k} \ell_{j} v_{j}\right|^{-2 \delta} \\
& \quad \times \exp \left\{\sqrt{-1}\left(\left|\frac{v_{0}+\sum_{j=1}^{k} \ell_{j} v_{j}}{2 \mathrm{e}^{h}}\right|^{2}-1\right)^{1 / 2} \times \frac{\theta \cdot\left(v_{0}+\sum_{j=1}^{k} \ell_{j} v_{j}\right)}{\left|v_{0}+\sum_{j=1}^{k} \ell_{j} v_{j}\right|}\right\} .
\end{aligned}
$$

On reconnaît ici une somme de Riemann, qui ne diffère de l'intégrale associée

$$
\begin{aligned}
I_{h}^{\prime}:=\left|v_{1} \wedge \cdots \wedge v_{k}\right|^{-1} \int_{\mathbb{R}^{k}} & 1_{\left\{|s|>2 \mathrm{e}^{h}\right\}} \cdot|s|^{-2 \delta} \\
& \quad \times \exp \left(\sqrt{-1}(\theta \cdot s) \times \frac{1}{2} \mathrm{e}^{-h}\left(1-\frac{4 \mathrm{e}^{2 h}}{|s|^{2}}\right)^{\frac{1}{2}}\right) \mathrm{d} s
\end{aligned}
$$

que par une quantité dominée par l'intégrale du module du gradient de la fonction intégrée, soit, la partie concernant le domaine $\left\{2 \mathrm{e}^{h}<|s|<2 \mathrm{e}^{h}+\mathrm{e}^{\frac{1}{2} h}\right\}$

BULletin DE LA SOCiÉtÉ MATHÉmATiQUe DE FRANCE 
étant négligeable, par

$$
\begin{aligned}
& I_{h}^{\prime \prime}:=\int_{\mathbb{R}^{k}} 1_{\left\{|s|>2 \mathrm{e}^{h}+\mathrm{e}^{\frac{1}{2} h}\right\}} \mid \nabla\left[\exp \left(\sqrt{-1}\left(\left|\frac{s+\mathcal{O}(1)}{2 \mathrm{e}^{h}}\right|^{2}-1\right)^{\frac{1}{2}} \frac{\theta \cdot(s+\mathcal{O}(1))}{|s+\mathcal{O}(1)|}\right)\right. \\
& \left.\times|s+\mathcal{O}(1)|^{-2 \delta}\right] \mid \mathrm{d} s \\
& =\int_{\mathbb{R}^{k}\left\{|s|>2 \mathrm{e}^{h}+\mathrm{e}^{\frac{1}{2} h}\right\}}\left(\mathcal{O}\left(\mathrm{e}^{-\frac{3}{4} h}\right) \cdot|s+\mathcal{O}(1)|^{-2 \delta}+\mathcal{O}\left(|s+\mathcal{O}(1)|^{-2 \delta-1}\right)\right) \mathrm{d} s \\
& =\mathcal{O}\left(\mathrm{e}^{-\frac{3}{4} h}\right) \int_{\mathbb{R}^{k}} 1_{\left\{|s|>2 \mathrm{e}^{h}+\mathrm{e}^{\frac{1}{2} h}\right\}} \cdot|s+\mathcal{O}(1)|^{-2 \delta} \mathrm{d} s=\mathcal{O}\left(\mathrm{e}^{\left(k-2 \delta-\frac{3}{4}\right) h}\right),
\end{aligned}
$$

qui sera finalement négligeable.

Un changement de variable donne ensuite

$$
I_{h}^{\prime}=\left(2 \mathrm{e}^{h}\right)^{k-2 \delta} \int_{\mathbb{R}^{k}}\left(1+|w|^{2}\right)^{\frac{1}{2} k-\delta-1}|w|^{2-k} \mathrm{e}^{\sqrt{-1}(\theta \cdot w)} \mathrm{d} w,
$$

de sorte qu'au total nous avons obtenu que

$$
\begin{aligned}
\Sigma_{h}^{\prime} & \sim\left|v_{1} \wedge \cdots \wedge v_{k}\right|^{-1}\left(2 \mathrm{e}^{h}\right)^{k-2 \delta} \int_{\mathbb{R}^{k}}\left(1+|w|^{2}\right)^{\frac{1}{2} k-\delta-1}|w|^{2-k} \mathrm{e}^{\sqrt{-1}(\theta \cdot w)} \mathrm{d} w \\
& =\operatorname{Covol}\left(\Gamma_{\infty}^{\prime}\right)^{-1} \mathrm{e}^{(k-2 \delta) h} 2^{k-2 \delta} \int_{\mathbb{R}^{k}} \mathrm{e}^{\sqrt{-1}(\pi(\theta) \cdot w)}\left(1+|w|^{2}\right)^{\frac{1}{2} k-\delta-1}|w|^{2-k} \mathrm{~d} w \\
& =: \mathrm{e}^{(k-2 \delta) h} \psi(|\pi(\theta)|),
\end{aligned}
$$

où $\pi(\theta)$ est la projection de $\theta$ sur $\mathbb{R}^{k}$ parallèlement à $\mathbb{R}^{d-k}$.

Par conséquent, nous avons pour tout $\varrho \in O\left(\mathbb{R}^{k}\right)$, par définition de $\Sigma_{h}^{\prime}$ et de $Y_{h}$ :

$$
\begin{aligned}
& \sum_{\gamma \in \varrho \Gamma_{\infty}^{\prime}} 1_{\left\{\ell_{h}\left(v_{-}, \gamma v_{+}\right)>0\right\}} \exp \left(\sqrt{-1} \theta \cdot \mathrm{e}^{-h} Y_{h}\left(v_{-}, \gamma v_{+}\right)\right) \mathrm{d}_{\infty}\left(v_{-}, \gamma v_{+}\right)^{-2 \delta} \\
& =\Sigma_{h}^{\prime}\left(\varrho^{-1} v_{-}, v_{+}, \varrho^{-1} \theta\right)=\mathrm{e}^{(k-2 \delta) h} \psi(|\pi(\theta)|)+o\left(\mathrm{e}^{(k-2 \delta) h}\right),
\end{aligned}
$$

et donc, puisque $\Gamma_{\infty} / \Gamma_{\infty}^{\prime} \subset O\left(\mathbb{R}^{k}\right)$, utilisant le corollaire 2 :

$$
\begin{aligned}
Z_{h}^{\theta}=\left|\chi_{h}\right|^{-1} \int_{\mathcal{D}^{2}}\left[\Gamma_{\infty}: \Gamma_{\infty}^{\prime}\right] \cdot\left(\mathrm{e}^{(k-2 \delta) h} \psi(|\pi(\theta)|)+o\left(\mathrm{e}^{(k-2 \delta) h}\right)\right) & \mathrm{d} \mu_{\infty}\left(v_{-}\right) \mathrm{d} \mu_{\infty}\left(v_{+}\right) \\
=C_{\mathcal{P}}^{-1} \frac{2 \delta-k}{2^{k-2 \delta} k} & \operatorname{Covol}\left(\Gamma_{\infty}\right)^{-1} \\
& \times 2^{k-2 \delta} \int_{\mathbb{R}^{k}} \mathrm{e}^{\sqrt{-1}(\pi(\theta) \cdot w)}\left(1+|w|^{2}\right)^{\frac{1}{2} k-\delta-1}|w|^{2-k} \mathrm{~d} w+o(1) \\
= & \pi^{-\frac{1}{2} k} \Gamma\left(\frac{1}{2} k\right)\left(\delta-\frac{1}{2} k\right) \int_{\mathbb{R}^{k}} \mathrm{e}^{\sqrt{-1}(\pi(\theta) \cdot w)}\left(1+|w|^{2}\right)^{\frac{1}{2} k-\delta-1}|w|^{2-k} \mathrm{~d} w+o(1) .
\end{aligned}
$$

TOME $130-2002-\mathrm{N}^{\mathrm{O}} 3$ 


\section{Cas des orbifolds hyperboliques complexes}

Nous supposons ici que $X=\mathbb{H}_{\mathbb{C}}^{d}$ est l'espace hyperbolique complexe de dimension $d$. Nos références sont surtout [1], [11], [13]. Voir aussi [9].

Nous utiliserons les deux modèles les plus classiques, celui de Siegel et le projectif :

$$
\begin{aligned}
\mathbb{H}_{\mathbb{C}}^{d} & =\left\{(w, z) \in \mathbb{C}^{d-1} \times\left.\mathbb{C}|2 \operatorname{Re}(z)>| w\right|^{2}\right\} \\
& =\left\{P\left(w, w_{1}, w_{0}\right) \in P \mathbb{C}^{d} \mid Q\left(w, w_{1}, w_{0}\right)<0\right\},
\end{aligned}
$$

où $Q\left(w, w_{1}, w_{0}\right):=|w|^{2}-w_{1} \bar{w}_{0}-w_{0} \bar{w}_{1}$, pour $\left(w, w_{1}, w_{0}\right) \in \mathbb{C}^{d-1} \times \mathbb{C} \times \mathbb{C}$. Le bord de $\mathbb{H}_{\mathbb{C}}^{d}$ est

$$
\partial \mathbb{H}_{\mathbb{C}}^{d}=\left\{(w, z) \in \mathbb{C}^{d-1} \times\left.\mathbb{C}|2 \operatorname{Re}(z)=| w\right|^{2}\right\} \cup\{\infty\} .
$$

Les coordonnées horosphériques de $(w, z) \in \overline{\mathbb{H}_{\mathbb{C}}^{d}} \backslash\{\infty\}$ sont $(\zeta, v, t) \in \mathbb{C}^{d-1} \times$ $\mathbb{R} \times \mathbb{R}_{+}$définies par

$$
\zeta=w, \quad v=-2 \operatorname{Im}(z), \quad t=2 \operatorname{Re}(z)-|w|^{2} .
$$

(Donc $z=\frac{1}{2}\left(t+|\zeta|^{2}-i v\right)$.)

La norme de Cygan est définie par

$$
\|(\zeta, v, t)\|:=\sqrt{\left.|t+| \zeta\right|^{2}-i v \mid} .
$$

Le groupe de Heisenberg $\mathcal{H}_{d}$ est $\mathbb{C}^{d-1} \times \mathbb{R}$ muni de la loi :

$$
(\zeta, v) \cdot\left(\zeta^{\prime}, v^{\prime}\right):=\left(\zeta+\zeta^{\prime}, v+v^{\prime}+2 \operatorname{Im}\left(\zeta \cdot \overline{\zeta^{\prime}}\right)\right) .
$$

On l'identifie à $\partial \mathbb{H}_{\mathbb{C}}^{d} \backslash\{\infty\}$ via $(\zeta, v) \equiv(\zeta, v, 0)$. Il conserve $\infty$ et agit sur $\overline{\mathbb{H}_{\mathbb{C}}^{d}} \backslash\{\infty\}$ par translation à gauche sur chaque horocycle basé en $\infty$ :

$$
(\zeta, v) \cdot\left(\zeta^{\prime}, v^{\prime}, t\right):=\left(\zeta+\zeta^{\prime}, v+v^{\prime}+2 \operatorname{Im}\left(\zeta \cdot \overline{\zeta^{\prime}}\right), t\right)
$$

Repassant aux coordonnées $(w, z)$ de Siegel, cela donne $(\zeta, v) \equiv\left(\zeta, \frac{1}{2}\left(|\zeta|^{2}-i v\right)\right)$ et

$$
\left.(\zeta, v) \cdot(w, z):=\left(w+\zeta, z+w \bar{\zeta}+\frac{1}{2}\left(|\zeta|^{2}-i v\right)\right)\right) .
$$

Le groupe unitaire $U(d-1)$ conserve $\infty$ et agit sur $\overline{\mathbb{H}_{\mathbb{C}}^{d}} \backslash\{\infty\}$ par rotation de la coordonnée $\zeta$. Le produit semi-direct $\mathcal{H}(d)$ des deux groupes $\mathcal{H}_{d}$ et $U(d-1)$ constitue le groupe des isométries de $\mathbb{H}_{\mathbb{C}}^{d}$ qui conservent l'orientation, $\infty$, et chaque horosphère $t=t_{0}$. Il contient en particulier le sous-groupe parabolique $\Gamma_{\xi}=\Gamma_{\infty}$ qui nous intéresse.

Utilisons une partie du théorème $[1,3.5]: \Gamma_{\infty}$ contient un sous-groupe normal d'indice fini, disons $\Gamma_{\infty}^{\prime}$, inclus dans $\mathcal{H}_{d}$. De plus $\Gamma_{\infty}$ agit librement et de façon cocompacte sur une variété affine $\mathcal{H}_{\Gamma}$ de $\partial \mathbb{H}_{\mathbb{C}}^{d} \backslash\{\infty\}$; nous noterons $\operatorname{Covol}\left(\Gamma_{\infty}\right)$ le volume de $\Gamma_{\infty} \backslash \mathcal{H}_{\Gamma}$.

Notons $k$ le rang de $\Gamma_{\infty}^{\prime}$ dans l'espace vectoriel réel $\mathbb{C}^{d-1} \times \mathbb{R} \equiv \mathbb{R}^{2 d-1}$, et fixons $V_{1}=\left(\zeta_{1}, v_{1}\right), \ldots, V_{k}=\left(\zeta_{k}, v_{k}\right) \in \Gamma_{\infty}^{\prime}$ qui engendrent le même sous-espace vectoriel que $\Gamma_{\infty}^{\prime}$. 
Alors tout $V \in \Gamma_{\infty}^{\prime}$ s'écrit $V=\sum_{j=1}^{k} r_{j} V_{j}$ (pour des réels $r_{j}$ ), et on a pour $n_{1}, \ldots, n_{k} \in \mathbb{Z}$

$$
V_{1}^{n_{1}} \cdot V_{k}^{n_{k}} \cdot V \cdot V_{k}^{n_{k}} \cdot \cdot V_{1}^{n_{1}}=\sum_{j=1}^{k}\left(r_{j}+2 n_{j}\right) V_{j} .
$$

Par conséquent le réseau (ou $\mathbb{Z}$-module libre) $\Gamma_{\infty}^{\prime \prime}$ engendré par $2 V_{1}, \ldots, 2 V_{k}$ constitue une partie d'indice fini de $\Gamma_{\infty}^{\prime}$, et donc de $\Gamma_{\infty}$.

De plus, si $\Gamma_{\infty}^{\prime} \subset \mathbb{C}^{d-1}$ alors $\Gamma_{\infty}^{\prime}$ est abélien et $\Gamma_{\infty}^{\prime \prime}$ est aussi un sous-groupe de $\Gamma_{\infty}$. Tandis que si $\Gamma_{\infty}^{\prime}$ n'est pas abélien, pour garantir que $\Gamma_{\infty}^{\prime \prime}$ est aussi un sous-groupe de $\Gamma_{\infty}$ il faut plutôt choisir $V_{1}$ engendrant $\Gamma_{\infty}^{\prime} \cap(\{0\} \times \mathbb{R})$ et le compléter par $2 V_{2}, \ldots, 2 V_{k}$; en effet, $\left[V_{i}, V_{j}\right] \in V_{1} \mathbb{Z}$ et $\left(2 V_{i}\right) \cdot\left(2 V_{j}\right)-2\left[V_{i}, V_{j}\right] \in \mathbb{C}^{d-1}$, ce qui fait que le $\mathbb{Z}$-module libre $\Gamma_{\infty}^{\prime \prime}$ engendré par $V_{1}, 2 V_{2}, \ldots, 2 V_{k}$ est bien un sous-groupe d'indice fini de $\Gamma_{\infty}$.

6.1. Croissance du sous-groupe $\Gamma_{\infty}$ : l'hypothèse $(*)$. — Fixons deux points $x=P(w, z, 1)$ et $x^{\prime}=P\left(w^{\prime}, z^{\prime}, 1\right)$ du même horocycle horizontal $t=1$. Dans les coordonnées projectives, l'élément $(\zeta, v) \in \mathcal{H}_{d}$ agit sur $x^{\prime}$ par

$$
(\zeta, v) \cdot x^{\prime}=P\left(\zeta+w^{\prime}, z^{\prime}+w^{\prime} \bar{\zeta}+\frac{1}{2}\left(|\zeta|^{2}-i v\right), 1\right) .
$$

Par conséquent nous avons (pour $x, x^{\prime}$ variant dans un compact de l'horocycle $\{t=1\})$ :

$$
\begin{aligned}
\operatorname{ch}^{2}\left[d\left(x,(\zeta, v) \cdot x^{\prime}\right)\right] & =\left|\frac{|\zeta|^{2}-i v}{2}+z^{\prime}+w^{\prime} \bar{\zeta}+\bar{z}-\bar{w}\left(\zeta+w^{\prime}\right)\right|^{2} \\
& =\left|\frac{|\zeta|^{2}}{2}+\mathcal{O}(\zeta)\right|^{2}+\left|\frac{v}{2}+\mathcal{O}(\zeta)\right|^{2} \\
& =\frac{1}{4}\left(\|(\zeta, v)\|^{4}+\mathcal{O}\left(|\zeta|^{3}+|\zeta| \cdot|v|\right)\right) \\
& =\frac{1}{4}\left(\|(\zeta, v)\|^{4}+\mathcal{O}\left(\|(\zeta, v)\|^{3}\right)\right)=\frac{1}{4}(\|(\zeta, v)\|+\mathcal{O}(1))^{4} .
\end{aligned}
$$

Corlette et Iozzi ont déjà noté (voir [4, preuve du lemme 3.5, formule (3.5)]) que $4 \operatorname{ch}^{2}[d(x,(\zeta, v) x)] \sim\|(\zeta, v)\|^{4}$; nous avons toutefois besoin du calcul légèrement plus précis ci-dessus, avec la possibilité que $x$ soit différent de $x^{\prime}$ et le contrôle de l'équivalent. Donc

$$
\left\{\gamma \in \Gamma_{\infty}^{\prime \prime} \mid d\left(x, \gamma x^{\prime}\right)<\varrho\right\}=\left\{(\zeta, v) \in \Gamma_{\infty}^{\prime \prime} ;\|(\zeta, v)\|<\sqrt{2 \operatorname{ch} \varrho}-\mathcal{O}(1)\right\}
$$

a même cardinal que

$$
\left\{\left(n_{1}, \ldots, n_{k}\right) \in \mathbb{Z}^{k} ;\left\|\sum_{j=1}^{k} 2 n_{j} V_{j}\right\|<\sqrt{2 \operatorname{ch} \varrho}-\mathcal{O}(1)\right\} .
$$

TOME $130-2002-\mathrm{N}^{\mathrm{O}} 3$ 
Fixons un isomorphisme $A$ de l'espace vectoriel réel $\mathcal{U}$ engendré par $\left(V_{1}, \ldots, V_{k}\right)$ tel que $\left(A V_{1}, \ldots, A V_{k}\right)$ soit orthonormé, et notons

$$
\varrho^{\prime}:=\sqrt{2 \operatorname{ch} \varrho}-\mathcal{O}(1)=\mathrm{e}^{\frac{1}{2} \varrho}-\mathcal{O}(1),
$$

Vol pour désigner le volume euclidien, Sur la surface euclidienne, et

$$
\mathcal{E}_{\varrho}:=\{V \in \mathcal{U} ;\|V\|<\varrho\} .
$$

Nous avons

$$
\begin{aligned}
& \operatorname{Card}\left\{\gamma \in \Gamma_{\infty}^{\prime \prime} \mid d\left(x, \gamma x^{\prime}\right)<\varrho\right\}=\operatorname{Card}\left\{n \in \mathbb{Z}^{k} ; \sum_{j=1}^{k} 2 n_{j} V_{j} \in \mathcal{E}_{\varrho^{\prime}}\right\} \\
& =\operatorname{Card}\left\{n \in \mathbb{Z}^{k} ; \sum_{j=1}^{k} n_{j} A V_{j} \in \frac{1}{2} A \mathcal{E}_{\varrho^{\prime}}\right\}=\operatorname{Vol}\left(\frac{1}{2} A \mathcal{E}_{\varrho^{\prime}}\right)+\mathcal{O}\left(\operatorname{Sur}\left(\frac{1}{2} A \mathcal{E}_{\varrho^{\prime}}\right)\right) \\
& =\operatorname{det}\left(\frac{1}{2} A\right) \operatorname{Vol}\left(\mathcal{E}_{\varrho^{\prime}}\right)+\mathcal{O}\left(\operatorname{Sur}\left(\mathcal{E}_{\varrho^{\prime}}\right)\right) \\
& =2^{-k}\left|V_{1} \wedge \cdots \wedge V_{k}\right|^{-1} \operatorname{Vol}\left(\mathcal{E}_{\varrho^{\prime}}\right)+\mathcal{O}\left(\operatorname{Sur}\left(\mathcal{E}_{\varrho^{\prime}}\right)\right) .
\end{aligned}
$$

Or, si $\Gamma_{\infty}^{\prime}$ n'est pas inclus dans $\mathbb{C}^{d-1}$ et si $k \neq 1$, notant $B^{k-1}$ la boule unité de $\mathbb{R}^{k-1}$ :

$$
\begin{aligned}
\operatorname{Vol}\left(\mathcal{E}_{\varrho}\right) & =2 \int_{0}^{\varrho^{2}} \operatorname{Vol}\left(B^{k-1}\right)\left(\left(\varrho^{4}-v^{2}\right)^{\frac{1}{4}}\right)^{k-1} \mathrm{~d} v \\
& =2 \operatorname{Vol}\left(B^{k-1}\right) \varrho^{k+1} \int_{0}^{1}\left(1-v^{2}\right)^{\frac{1}{4}(k-1)} \mathrm{d} v, \\
\int_{0}^{1}\left(1-v^{2}\right)^{\frac{1}{4}(k-1)} \mathrm{d} v & =\frac{1}{2} \int_{0}^{1} u^{\frac{1}{4}(k-1)}(1-u)^{-\frac{1}{2}} \mathrm{~d} u=\frac{1}{2} B\left(\frac{1}{4}(k+3), \frac{1}{2}\right) .
\end{aligned}
$$

Et si $\Gamma_{\infty}^{\prime}$ est inclus dans $\mathbb{C}^{d-1}$ :

$$
\operatorname{Vol}\left(\mathcal{E}_{\varrho}\right)=\operatorname{Vol}\left(B^{k}\right) \varrho^{k} .
$$

Donc si $\Gamma_{\infty}^{\prime}$ n'est pas inclus dans $\mathbb{C}^{d-1}$ et si $k \neq 1$ :

$$
\begin{aligned}
\operatorname{Card}\{\gamma & \left.\in \Gamma_{\infty}^{\prime \prime} \mid d\left(x, \gamma x^{\prime}\right)<\varrho\right\} \\
& =2^{-k}\left|V_{1} \wedge \cdots \wedge V_{k}\right|^{-1} \operatorname{Vol}\left(B^{k-1}\right) B\left(\frac{1}{4}(k+3), \frac{1}{2}\right) \varrho^{\prime k+1}+\mathcal{O}\left(\varrho^{\prime k}\right) \\
& =\frac{\operatorname{Vol}\left(B^{k-1}\right) B\left(\frac{1}{4}(k+3), \frac{1}{2}\right)}{\operatorname{Covol}\left(\Gamma_{\infty}^{\prime \prime}\right)} \exp \left(\frac{1}{2}(k+1) \varrho\right)+\mathcal{O}\left(\mathrm{e}^{\frac{1}{2} k \varrho}\right) .
\end{aligned}
$$

De là on déduit comme dans le cas réel que, $k$ étant le rang de $\Gamma_{\infty}^{\prime}$, on a :

$\operatorname{Card}\left\{\gamma \in \Gamma_{\infty} \mid d\left(x, \gamma x^{\prime}\right)<\varrho\right\}=\frac{\operatorname{Vol}\left(B^{k-1}\right) B\left(\frac{1}{4}(k+3), \frac{1}{2}\right)}{\operatorname{Covol}\left(\Gamma_{\infty}\right)} \mathrm{e}^{\frac{1}{2}(k+1) \varrho}+\mathcal{O}\left(\mathrm{e}^{\frac{1}{2} k \varrho}\right)$, BULLETIN DE LA SOCIÉTÉ MATHÉMATIQUE DE FRANCE 
toujours si $\Gamma_{\infty}^{\prime}$ n'est pas inclus dans $\mathbb{C}^{d-1}$ et si $k \neq 1$. Tandis que si $\Gamma_{\infty}^{\prime}$ est inclus dans $\mathbb{C}^{d-1}$ :

$$
\operatorname{Card}\left\{\gamma \in \Gamma_{\infty} \mid d\left(x, \gamma x^{\prime}\right)<\varrho\right\}=\frac{\operatorname{Vol}\left(B^{k}\right)}{\operatorname{Covol}\left(\Gamma_{\infty}\right)} \mathrm{e}^{\frac{1}{2} k \varrho}+\mathcal{O}\left(\mathrm{e}^{\frac{1}{2}(k-1) \varrho}\right) .
$$

Enfin si $\Gamma_{\infty}^{\prime} \not \subset \mathbb{C}^{d-1} \cup \mathbb{R}$ et $k=1$, on voit directement que

$$
\text { Card }\left\{n \in \mathbb{Z} ;\|2 n(\zeta, v)\|<\varrho^{\prime}\right\}=\frac{\varrho^{\prime}}{|\zeta|}+\mathcal{O}(1),
$$

d'où $\delta_{\infty}=\frac{1}{2}$ et $C(x, y) \equiv \operatorname{Covol}\left(\pi\left(\Gamma_{\infty}\right)\right)^{-1}, \pi$ étant la projection de $\mathbb{C}^{d-1} \times \mathbb{R}$ $\operatorname{sur} \mathbb{C}^{d-1}$; et si $\Gamma_{\infty}^{\prime} \subset \mathbb{R}$ on obtient $\delta_{\infty}=1$ et $C(x, y) \equiv \operatorname{Covol}\left(\Gamma_{\infty}\right)^{-1}$.

C'est dire en particulier que l'hypothèse $\left(^{*}\right)$ est bien vérifiée; nous venons pour résumer d'établir ( $k$ désignant le rang de $\Gamma_{\xi}^{\prime}$ et $\pi$ la projection de $\mathbb{C}^{d-1} \times \mathbb{R}$ sur $\left.\mathbb{C}^{d-1}\right)$ :

Proposition 7 . - Nous avons localement uniformément par rapport à $x, y \in$ $H_{0}$ :

$$
\operatorname{Card}\left\{\gamma \in \Gamma_{\xi} \mid d(x, \gamma y) \leq \varrho\right\}=C_{\mathcal{P}} \mathrm{e}^{\delta_{\xi} \varrho}+\mathcal{O}\left(\mathrm{e}^{\left(\delta_{\xi}-\frac{1}{2}\right) \varrho}\right)
$$

lorsque $\varrho \rightarrow+\infty$ avec

1) si $\Gamma_{\xi}^{\prime} \not \subset \mathbb{C}^{d-1}$ et $k \neq 1: \delta_{\xi}=\frac{1}{2}(k+1)$ et

$$
C_{\mathcal{P}}=\operatorname{Vol}\left(B^{k-1}\right) B\left(\frac{1}{4}(k+3), \frac{1}{2}\right) / \operatorname{Covol}\left(\Gamma_{\xi}\right) ;
$$

2) si $\Gamma_{\xi}^{\prime} \subset \mathbb{C}^{d-1}$ : $\delta_{\xi}=\frac{1}{2} k$ et $C_{\mathcal{P}}=\operatorname{Vol}\left(B^{k}\right) / \operatorname{Covol}\left(\Gamma_{\xi}\right)$;

3) si $\Gamma_{\xi}^{\prime} \subset \mathbb{R}: \delta_{\xi}=1$ et $C_{\mathcal{P}}=\operatorname{Covol}\left(\Gamma_{\xi}\right)^{-1}$;

4) si $\Gamma_{\xi}^{\prime} \not \subset \mathbb{C}^{d-1} \cup \mathbb{R}$ et $k=1: \delta_{\xi}=\frac{1}{2}$ et $C_{\mathcal{P}}=\operatorname{Covol}\left(\pi\left(\Gamma_{\xi}\right)\right)^{-1}$.

6.2. Calcul de la fonction $\boldsymbol{\ell}_{\boldsymbol{h}}$. - Soient $(\zeta, v) \in \mathcal{H}_{d} \backslash\{0\}$ et

$$
M:=\left(\begin{array}{ccc}
I & \zeta & 0 \\
0 & \frac{1}{2}\left(|\zeta|^{2}-i v\right) & 0 \\
2^{t} \bar{\zeta}\left(|\zeta|^{2}+i v\right)^{-1} & 1 & 2\left(|\zeta|^{2}+i v\right)^{-1}
\end{array}\right) .
$$

C'est une matrice de format $(d+1) \times(d+1)$ qui préserve la forme quadratique $Q$; elle représente donc une isométrie (conservant l'orientation) de $\mathbb{H}_{\mathbb{C}}^{d}$. De plus elle envoie $0=P(0,0,1)$ sur lui-même et $\infty=P(0,1,0)$ $\operatorname{sur}(\zeta, v) \equiv\left(\zeta, \frac{1}{2}|\zeta|^{2}-i v\right)=P\left(\zeta, \frac{1}{2}|\zeta|^{2}-i v, 1\right)$, et donc la géodésique $\overrightarrow{0 \infty}$ sur la géodésique $\overrightarrow{0(\zeta, v)}$. Comme $\left(s \mapsto\left(0,2 \mathrm{e}^{2 s}\right)=P\left(0,2 \mathrm{e}^{2 s}, 1\right)\right)$ paramètre la géodésique $\overrightarrow{0 \infty}$, on voit que la géodésique $\overrightarrow{0(\zeta, v)}$ est paramétrée par $s \mapsto P\left(2 \mathrm{e}^{2 s} \zeta,\left(|\zeta|^{2}-i v\right) \mathrm{e}^{2 s}, 2\left(|\zeta|^{2}+i v\right)^{-1}+2 \mathrm{e}^{2 s}\right)$.

Elle intersecte l'horocycle horizontal de hauteur $t$ pour les valeurs du paramètre $s$ résolvant :

$2 \operatorname{Re}\left(\left(|\zeta|^{2}-i v\right) \mathrm{e}^{2 s}\left[2\left(|\zeta|^{2}-i v\right)^{-1}+2 \mathrm{e}^{2 s}\right]\right)-\left|2 \mathrm{e}^{2 s} \zeta\right|^{2}=t \cdot\left|2\left(|\zeta|^{2}+i v\right)^{-1}+2 \mathrm{e}^{2 s}\right|^{2}$ 


$$
\Longleftrightarrow\|(\zeta, v)\|^{4} t \mathrm{e}^{4 s}-\left(\|(\zeta, v)\|^{4}-2|\zeta|^{2} t\right) \mathrm{e}^{2 s}+t=0 .
$$

Notons par commodité $N:=\|(\zeta, v)\|$. L'équation ci-dessus a des solutions si et seulement si

$$
\left.0 \leq N^{8}-4 N^{4}|\zeta|^{2} t-4 v^{2} t^{2}=\left(N^{4}-2\left(N^{2}+|\zeta|^{2}\right) t\right)\left(N^{4}+2\left(N^{2}-|\zeta|^{2}\right) t\right)\right),
$$

c'est-à-dire

$$
\frac{N^{4}}{N^{2}+|\zeta|^{2}}=\frac{|\zeta|^{4}+|v|^{2}}{\sqrt{|\zeta|^{4}+|v|^{2}}+|\zeta|^{2}} \geq 2 t
$$

Et dans ce cas, nous avons la valeur explicite de la longeur de la géodésique $\overrightarrow{0(\zeta, v)}$ située au-dessus de l'horocycle horizontal de hauteur $t=\mathrm{e}^{2 h}$ :

$$
\ell_{h}(0,(\zeta, v))=\log \left[\frac{N^{4}-2|\zeta|^{2} t+\sqrt{N^{8}-4 N^{4}|\zeta|^{2} t-4 v^{2} t^{2}}}{2 N^{2} t}\right] .
$$

Pour évaluer les séries de Poincaré généralisées et en particulier la masse asymptotique de la pointe, nous avons besoin de la quantité $\ell_{h}\left(\left(\zeta_{-}, v_{-}\right),(\zeta, v)\right.$. $\left.\left(\zeta_{+}, v_{+}\right)\right)$, relative à une géodésique $\overrightarrow{\left(\zeta_{-}, v_{-}\right)(\zeta, v) \cdot\left(\zeta_{+}, v_{+}\right)}$, pour $\left(\zeta_{-}, v_{-}\right)$et $\left(\zeta_{+}, v_{+}\right)$variant dans un compact de $\mathcal{H}_{d}$ et $(\zeta, v)$ variant dans un sous-groupe parabolique. Or

$$
\begin{aligned}
\ell_{h}\left(\left(\zeta_{-}, v_{-}\right),(\zeta, v) \cdot\left(\zeta_{+}, v_{+}\right)\right) & =\ell_{h}\left(0,(\zeta, v) \cdot\left(\zeta_{+}, v_{+}\right) \cdot\left(\zeta_{-}, v_{-}\right)^{-1}\right) \\
& =\ell_{h}\left(0,(\zeta, v) \cdot\left(\zeta_{0}, v_{0}\right)\right)
\end{aligned}
$$

$\operatorname{avec}\left(\zeta_{0}, v_{0}\right):=\left(\zeta_{+}, v_{+}\right) \cdot\left(\zeta_{-}, v_{-}\right)^{-1}$ variant dans un compact de $\mathcal{H}_{d}$.

Il suffit donc de remplacer dans l'expression explicite ci-dessus $(\zeta, v)$ par $\left(\zeta+\zeta_{0}, v+v_{0}+2 \operatorname{Im}\left(\zeta \cdot \overline{\zeta_{0}}\right)\right)$. Remarquons d'abord que nous avons

$$
\left\|\left(\zeta+\zeta_{0}, v+v_{0}+2 \operatorname{Im}\left(\zeta \overline{\zeta_{0}}\right)\right)\right\|=\|(\zeta, v)\|+\mathcal{O}(1)
$$

lorsque $(\zeta, v) \rightarrow \infty$, id est lorsque $N \rightarrow \infty$.

Nous avons de même $\left|\zeta+\zeta_{0}\right|=|\zeta|+\mathcal{O}(1)$ et $\left|v+v_{0}+2 \operatorname{Im}\left(\zeta \cdot \overline{\zeta_{0}}\right)\right|=|v|+\mathcal{O}(N)$.

En particulier la condition d'intersection avec l'horocycle horizontal $H_{h}$ peut s'écrire :

$$
2 \mathrm{e}^{2 h} \leq \frac{(N+\mathcal{O}(1))^{4}}{(N+\mathcal{O}(1))^{2}+(|\zeta|+\mathcal{O}(1))^{2}}=\frac{N^{4}}{N^{2}+|\zeta|^{2}}(1+\mathcal{O}(1 / N)),
$$

ou bien encore a fortiori

$$
(\zeta, v) \in \mathcal{E}_{h}:=\left\{(\zeta, v) \in \mathcal{H}_{d} \mid 2 \mathrm{e}^{2 h}\left(1+\mathcal{O}\left(\mathrm{e}^{-h}\right)\right) \leq N^{4} /\left(N^{2}+|\zeta|^{2}\right)\right\} .
$$

De plus, notant $\mathcal{O}$ pour $\mathcal{O}(1)$, nous avons lorsque cette condition est réalisée :

$$
\begin{array}{r}
\exp \left[\ell_{h}\left(0,(\zeta, v) \cdot\left(\zeta_{0}, v_{0}\right)\right)\right] \\
=\frac{\mathrm{e}^{-2 h}}{2}\left(N^{2}-2|\zeta|^{2} N^{-2} \mathrm{e}^{2 h}+\sqrt{N^{4}-4|\zeta|^{2} \mathrm{e}^{2 h}-4 v^{2} N^{-4} \mathrm{e}^{4 h}+\mathcal{O}\left(N \mathrm{e}^{2 h}\right)}\right) \\
\times(1+\mathcal{O}(1 / N)),
\end{array}
$$

BULLETIN DE LA SOCIÉTÉ MATHÉMATIQUe DE FRANCE 
utilisant qu'on a $\operatorname{sur}\left\{2 \mathrm{e}^{2 h} \leq N^{4} /\left(N^{2}+|\zeta|^{2}\right)(1+\mathcal{O}(1 / N))\right\}: \mathrm{e}^{h}=\mathcal{O}(N)$ et $\left|\frac{N^{3}}{N^{4}-2|\zeta|^{2} \mathrm{e}^{2 h}}\right| \leq N^{-1}\left(1-\frac{|\zeta|^{2}}{N^{2}+|\zeta|^{2}}(1+\mathcal{O}(1 / N))\right)^{-1} \leq 2 N^{-1}+\mathcal{O}\left(N^{-2}\right)$.

Pour contrôler le gradient de $\ell_{h}^{0}$ (qui comporte une racine carrée pouvant s'annuler), nous avons besoin de nous restreindre aux géodésiques dépassant le niveau $\mathrm{e}^{h}$; il suffira de dépasser par exemple $\mathrm{e}^{h}+1$. Considérons donc

$$
\mathcal{E}_{h}^{\prime}:=\left\{(\zeta, v) \in \mathcal{H}_{d} \mid 2 \mathrm{e}^{2 h}\left(1+\mathrm{e}^{-h}\right) \leq N^{4} /\left(N^{2}+|\zeta|^{2}\right)\right\}
$$

puis $\mathcal{E}_{h}^{\prime \prime}:=\mathcal{E}_{h} \backslash \mathcal{E}_{h}^{\prime}$. Sur $\mathcal{E}_{h}^{\prime}$, nous avons d'une part

$$
N^{4} \geq 2\left(N^{2}+|\zeta|^{2}\right) \mathrm{e}^{2 h} \Longrightarrow N^{4} \geq 4|\zeta|^{2} \mathrm{e}^{2 h} \Longrightarrow N^{2}-2|\zeta|^{2} N^{-2} \mathrm{e}^{2 h} \geq \frac{1}{2} N^{2},
$$

et d'autre part

$$
N^{4}-4|\zeta|^{2} \mathrm{e}^{2 h}-4 v^{2} N^{-4} \mathrm{e}^{4 h} \geq 2 N^{2} \mathrm{e}^{h},
$$

d'où

$$
\begin{gathered}
\left|\frac{\sqrt{N^{4}-4|\zeta|^{2} \mathrm{e}^{2 h}-4 v^{2} N^{-4} \mathrm{e}^{4 h}+\mathcal{O}\left(N \mathrm{e}^{2 h}\right)}-\sqrt{N^{4}-4|\zeta|^{2} \mathrm{e}^{2 h}-4 v^{2} N^{-4} \mathrm{e}^{4 h}}}{N^{2}-2|\zeta|^{2} N^{-2} \mathrm{e}^{2 h}}\right| \\
\leq \frac{\left|\mathcal{O}\left(N \mathrm{e}^{2 h}\right)\right|}{\mathrm{e}^{2 h} \sqrt{N^{4}-4|\zeta|^{2} \mathrm{e}^{2 h}}}=\mathcal{O}\left(\mathrm{e}^{-\frac{1}{2} h}\right) .
\end{gathered}
$$

Par conséquent, nous avons sur $\mathcal{E}_{h}^{\prime}$ :

$$
\begin{aligned}
& \ell_{h}^{0}(\zeta, v):=\ell_{h}\left(0,(\zeta, v) \cdot\left(\zeta_{0}, v_{0}\right)\right) \\
&=\log \left(N^{2} \mathrm{e}^{-2 h}-2|\zeta|^{2} N^{-2}+\mathrm{e}^{-2 h} \sqrt{N^{4}-4|\zeta|^{2} \mathrm{e}^{2 h}-4 v^{2} N^{-4} \mathrm{e}^{4 h}}\right) \\
&-\log 2+\mathcal{O}\left(\mathrm{e}^{-\frac{1}{2} h}\right) .
\end{aligned}
$$

Enfin nous avons les majorations immédiates :

$$
0 \leq \ell_{h}^{0} \leq 2 \log \left(N \mathrm{e}^{-h}\right)+\mathcal{O}\left(\mathrm{e}^{-h}\right) \text { sur } \mathcal{E}_{h}
$$

et $N=\mathcal{O}\left(\mathrm{e}^{h}\right)$ sur $\mathcal{E}_{h}^{\prime \prime}$, qui entraînent que $\ell_{h}^{0}$ est bornée sur $\mathcal{E}_{h}^{\prime \prime}$.

6.3. Asymptotique des séries de Poincaré généralisées. - Voici le résultat qui précise la proposition 1 dans le cas hyperbolique complexe :

Proposition 8. - Considérons pour tout $h>0$ la série de Poincaré généralisée

$\Sigma_{h}:=\int_{\mathcal{D}^{2}} \sum_{\gamma \in \Gamma_{\xi}}\left(1_{] 0,+\infty[} \times \phi\right) \circ \ell_{h}\left(V_{-}, \gamma V_{+}\right) \times\left(d_{\xi}\left(V_{-}, \gamma V_{+}\right)\right)^{-2 \delta} \mathrm{d} \mu_{\xi}\left(V_{-}\right) \mathrm{d} \mu_{\xi}\left(V_{+}\right)$, où $\phi$ est une fonction de classe $C^{1}$ de $\mathbb{R}_{+}$dans $\mathbb{R}$ telle que

$$
\int_{0}^{\infty} \phi_{1}^{\prime}(t) \mathrm{e}^{-\left(\delta-\delta_{\xi}\right) t} \mathrm{~d} t<\infty
$$

TOME $130-2002-\mathrm{N}^{\mathrm{O}} 3$ 
avec $\phi_{1}^{\prime}(t):=\sup \left|\phi^{\prime}\right|\left(\left[(t-1)^{+}, t+1\right]\right)$. Alors nous avons lorsque $h \rightarrow \infty$ :

$$
\Sigma_{h}=\lambda_{\mathcal{P}}^{2} K_{\mathcal{P}} \operatorname{Covol}\left(\Gamma_{\xi}\right)^{-1} \exp \left(-2\left(\delta-\delta_{\xi}\right) h\right)\left(1+\mathcal{O}\left(\mathrm{e}^{-\frac{1}{2} h}\right)\right),
$$

où l'on a ( $k$ étant toujours le rang de $\left.\Gamma_{\xi}^{\prime}\right)$ :

1) si $\Gamma_{\xi}^{\prime} \not \subset \mathbb{C}^{d-1}$ et $k \neq 1: \delta_{\xi}=\frac{1}{2}(k+1)$ et $K_{\mathcal{P}}=2^{\frac{1}{2}(k+1)} \operatorname{Vol}\left(\mathbb{S}^{k-2}\right) \int_{0}^{\infty} J \phi$, où

$$
J(\tau):=(\operatorname{sh} \tau) \int_{0}^{\infty}(1+(\operatorname{ch} \tau) \operatorname{ch} u)^{\frac{1}{2}(k-1)-\delta}(\operatorname{ch} u)^{\delta-k+1} \mathrm{~d} u ;
$$

2) si $\Gamma_{\xi}^{\prime} \subset \mathbb{C}^{d-1}: \delta_{\xi}=\frac{1}{2} k$ et

$$
K_{\mathcal{P}}=2^{k-1-\delta} \operatorname{Vol}\left(\mathbb{S}^{k-1}\right) \int_{0}^{\infty}\left(\operatorname{ch} \frac{1}{2} t\right)^{k-1-2 \delta}\left(\operatorname{sh} \frac{1}{2} t\right) \phi(t) \mathrm{d} t
$$

3) si $\Gamma_{\xi}^{\prime} \subset \mathbb{R}: \delta_{\xi}=1$ et $K_{\mathcal{P}}=4 \int_{0}^{\infty}(\operatorname{ch} \tau)^{-\delta}(\operatorname{sh} \tau) \phi(\tau) d \tau$;

4) si $\Gamma_{\xi}^{\prime} \not \subset \mathbb{C}^{d-1} \cup \mathbb{R}$ et $k=1: \delta_{\xi}=\frac{1}{2}$ et

$$
K_{\mathcal{P}}=\frac{2^{1-\delta} \operatorname{Covol}\left(\Gamma_{\xi}\right)}{\operatorname{Covol}\left(\pi\left(\Gamma_{\xi}\right)\right)} \int_{0}^{\infty}\left(\operatorname{ch} \frac{1}{2} t\right)^{-2 \delta}\left(\operatorname{sh} \frac{1}{2} t\right) \phi(t) \mathrm{d} t
$$

$\pi$ étant la projection de $\mathbb{C}^{d-1} \times \mathbb{R}$ sur $\mathbb{C}^{d-1}$.

REMARQUe. - Le cas 2) est analogue au cas hyperbolique réel.

Démonstration. - Il suffit bien sûr de considérer le cas où $\xi=\infty$. Nous devons étudier la somme :

$$
\Sigma_{h}^{\prime}:=\sum_{\gamma \in \Gamma_{\infty}}\left(1_{0,+\infty[} \times \phi\right) \circ \ell_{h}\left(0, \gamma \cdot\left(\zeta_{0}, v_{0}\right)\right)\left(d_{\infty}\left(0, \gamma \cdot\left(\zeta_{0}, v_{0}\right)\right)\right)^{-2 \delta} .
$$

Utilisant [13, prop. 3.8], nous avons, notant toujours $N:=\|(\zeta, v)\|$ :

$$
\begin{aligned}
d_{\infty}\left(\left(\zeta_{-}, v_{-}\right),(\zeta, v) \cdot\left(\zeta_{+}, v_{+}\right)\right) & =d_{\infty}\left(0,(\zeta, v) \cdot\left(\zeta_{0}, v_{0}\right)\right) \\
& =\left\|\left(\zeta+\zeta_{0}, v+v_{0}+2 \operatorname{Im}\left(\zeta \cdot \overline{\zeta_{0}}\right)\right)\right\| / \sqrt{2} \\
& =\|(\zeta, v)\| / \sqrt{2}+\mathcal{O}(1)=\frac{N}{\sqrt{2}}(1+\mathcal{O}(1 / N))
\end{aligned}
$$

Observons que tout élément de $U(d-1)$ conserve d'une part la norme de Cygan et donc la distance de Hamenstädt $d_{\infty}$, et d'autre part la fonction $\ell_{h}(0, \cdot)$. Nous avons donc

$$
\begin{aligned}
\Sigma_{h}^{\prime}=\left[\Gamma_{\infty}, \Gamma_{\infty}^{\prime}\right] \sum_{\gamma \in \Gamma_{\infty}^{\prime}}\left(1_{] 0,+\infty[} \times \phi\right) \circ \ell_{h}\left(0, \gamma \cdot\left(\zeta_{0}, v_{0}\right)\right)\left(d_{\infty}\left(0, \gamma \cdot\left(\zeta_{0}, v_{0}\right)\right)\right)^{-2 \delta} \\
=\left[\Gamma_{\infty}, \Gamma_{\infty}^{\prime}\right] \sum_{(\zeta, v) \in \Gamma_{\infty}^{\prime}}\left(1_{] 0,+\infty[} \times \phi\right) \circ \ell_{h}\left(0,(\zeta, v) \cdot\left(\zeta_{0}, v_{0}\right)\right) \\
\times(N / \sqrt{2})^{-2 \delta}(1+\mathcal{O}(1 / N)) .
\end{aligned}
$$

BULLETIN DE LA SOCiÉTÉ MATHÉMATIQUe DE FRANCE 
Notons comme plus haut (dans la section 6.2),

$$
\begin{aligned}
\ell_{h}^{0}(\zeta, v) & :=\ell_{h}\left(0,(\zeta, v) \cdot\left(\zeta_{0}, v_{0}\right)\right), \\
\mathcal{E}_{h} & :=\left\{(\zeta, v) \in \mathcal{H}_{d} \mid 2 \mathrm{e}^{2 h}\left(1+\mathcal{O}\left(\mathrm{e}^{-h}\right)\right) \leq N^{4} /\left(N^{2}+|\zeta|^{2}\right)\right\} \\
\mathcal{E}_{h}^{\prime} & :=\left\{(\zeta, v) \in \mathcal{H}_{d} \mid 2 \mathrm{e}^{2 h}\left(1+\mathrm{e}^{-h}\right) \leq N^{4} /\left(N^{2}+|\zeta|^{2}\right)\right\},
\end{aligned}
$$

puis $\mathcal{E}_{h}^{\prime \prime}:=\mathcal{E}_{h} \backslash \mathcal{E}_{h}^{\prime}$.

Par définition de $\Gamma_{\infty}^{\prime \prime}$, tout élément de $\Gamma_{\infty}^{\prime}$ est à distance bornée d'un élément de $\Gamma_{\infty}^{\prime \prime}$, et donc pour tout $(\zeta, v) \in \Gamma_{\infty}^{\prime}$ il existe $\left(\zeta^{\prime}, v^{\prime}\right) \in \Gamma_{\infty}^{\prime \prime}$ tel que $(\zeta, v)\left(\zeta_{0}, v_{0}\right)=\left(\zeta^{\prime}, v^{\prime}\right)\left(\zeta_{0}^{\prime}, v_{0}^{\prime}\right),\left(\zeta_{0}^{\prime}, v_{0}^{\prime}\right)$ variant dans un compact de $\mathcal{H}_{d}$ (de même que $\left.\left(\zeta_{0}, v_{0}\right)\right)$. De sorte que nous avons :

$$
\begin{aligned}
\Sigma_{h}^{\prime} & =\left[\Gamma_{\infty}, \Gamma_{\infty}^{\prime \prime}\right] 2^{\delta}\left(1+\mathcal{O}\left(\mathrm{e}^{-h}\right)\right) \sum_{(\zeta, v) \in \Gamma_{\infty}^{\prime \prime} \cap \mathcal{E}_{h}} \phi \circ \ell_{h}^{0}(\zeta, v) N^{-2 \delta} \\
& =\left[\Gamma_{\infty}, \Gamma_{\infty}^{\prime \prime}\right] 2^{\delta}\left(1+\mathcal{O}\left(\mathrm{e}^{-h}\right)\right) \sum_{n \in \mathbb{Z}^{k}}\left(1_{\mathcal{E}_{h}}\left(\phi \circ \ell_{h}^{0}\right)\right)\left(\sum_{j=1}^{k} 2 n_{j} V_{j}\right)\left\|\sum_{j=1}^{k} 2 n_{j} V_{j}\right\|^{-2 \delta}
\end{aligned}
$$

par définition de $\Gamma_{\infty}^{\prime \prime}$.

Réglons d'abord le cas de la contribution relative à $\mathcal{E}_{h}^{\prime \prime}$. Nous avons observé à la fin de la section précédente que $\ell_{h}^{0}$ est bornée sur cette partie, et donc nous avons :

$$
\begin{aligned}
\sum_{n \in \mathbb{Z}^{k}} & \left(1_{\mathcal{E}_{h}^{\prime \prime}} \times\left(\phi \circ \ell_{h}^{0}\right)\right)\left(\sum_{j=1}^{k} 2 n_{j} V_{j}\right)\left\|\sum_{j=1}^{k} 2 n_{j} V_{j}\right\|^{-2 \delta} \\
= & \mathcal{O}\left(\sum_{n \in \mathbb{Z}^{k}} 1_{\left\{\sum_{j=1}^{k} 2 n_{j} V_{j} \in \mathcal{E}_{h}^{\prime \prime}\right\}} \cdot\left\|\sum_{j=1}^{k} 2 n_{j} V_{j}\right\|^{-2 \delta}\right) \\
\leq & \operatorname{Card}\left\{n \in \mathbb{Z}^{k} ; \sum_{j=1}^{k} 2 n_{j} V_{j} \in \mathcal{E}_{h}\right\} \times \mathcal{O}\left(\mathrm{e}^{-2 \delta h}\right) \\
= & \mathcal{O}\left(\mathrm{e}^{\delta_{\infty} h-2 \delta h}\right)=o\left(\mathrm{e}^{-2\left(\delta-\delta_{\infty}\right) h}\right),
\end{aligned}
$$

où nous avons utilisé que $\mathrm{e}^{h}=\mathcal{O}(N)$ sur $\mathcal{E}_{h}$, et la proposition 7 (voir la section $6.1)$.

Revenons à la contribution déterminante, relative à $\mathcal{E}_{h}^{\prime}$. Nous avons

$$
\begin{aligned}
& \left|\sum_{n \in \mathbb{Z}^{k}}\left(1_{\mathcal{E}_{h}^{\prime}} \times F\right)\left(\sum_{j=1}^{k} 2 n_{j} V_{j}\right)-\int_{\mathbb{R}^{k}}\left(1_{\mathcal{E}_{h}^{\prime}} \times F\right)\left(\sum_{j=1}^{k} 2 s_{j} V_{j}\right) \mathrm{d} s\right| \\
& \quad \leq \int_{\mathbb{R}^{k}} 1_{\mathcal{E}_{h}^{\prime}}\left(\sum_{j=1}^{k} 2 n_{j} V_{j}\right) \sup |\nabla F|\left[\left\{\sum_{j=1}^{k} 2\left(n_{j}+s_{j}\right) V_{j} \mid 0 \leq s_{1}, \ldots, s_{k}<1\right\}\right],
\end{aligned}
$$

TOME $130-2002-\mathrm{N}^{\mathrm{O}} 3$ 
où $F(\zeta, v):=\phi \circ \ell_{h}^{0}(\zeta, v) \times\left(|\zeta|^{4}+v^{2}\right)^{-\frac{1}{2} \delta}$. Or $\nabla N=\mathcal{O}(1)$, et donc

$$
\nabla \ell_{h}^{0}(\zeta, v)=\mathcal{O}\left(\mathrm{e}^{-\frac{1}{2} h}\right)
$$

où nous avons utilisé l'observation (déjà faite à la section 6.2) que sur $\mathcal{E}_{h}^{\prime}$, on a $\mathrm{e}^{h} \leq N, N^{2}-2|\zeta|^{2} N^{-2} \mathrm{e}^{2 h} \geq N^{2} / 2$ et $N^{4}-4|\zeta|^{2} \mathrm{e}^{2 h}-4 v^{2} N^{-4} \mathrm{e}^{4 h} \geq 2 N^{2} \mathrm{e}^{h}$.

Par conséquent nous avons

$$
|\nabla F|=\left(\left|\phi^{\prime}\right|+|\phi|\right) \circ \ell_{h}^{0} \times N^{-2 \delta} \times \mathcal{O}\left(\mathrm{e}^{-\frac{1}{2} h}\right) \quad \text { sur } \quad \mathcal{E}_{h}^{\prime} .
$$

Par ailleurs, si $k \neq 1$ et $\Gamma_{\infty}^{\prime \prime} \not \subset \mathbb{C}^{d-1}$ (ce qui équivaut à $\Gamma_{\infty}^{\prime} \not \subset \mathbb{C}^{d-1}$ ), nous avons :

$$
\int_{\mathbb{R}^{k}}\left(1_{\mathcal{E}_{h}^{\prime}} \times F\right)\left(\sum_{j=1}^{k} 2 s_{j} V_{j}\right) \mathrm{d} s=\operatorname{Covol}\left(\Gamma_{\infty}^{\prime \prime}\right)^{-1} \int_{\mathbb{R}^{k-1} \times \mathbb{R}}\left(1_{\mathcal{E}_{h}^{\prime}} \times F\right)(x, v) \mathrm{d} x \mathrm{~d} v .
$$

Après remplacement de $\ell_{h}^{0}$ sur $\mathcal{E}_{h}^{\prime}$ par son expression trouvée à la fin de la section 6.2, suivi de quelques changements de variables élémentaires, nous obtenons

$$
\begin{aligned}
& \int_{\mathbb{R}^{k-1} \times \mathbb{R}}\left(1_{\mathcal{E}_{h}^{\prime}} \times F\right)(x, v) \mathrm{d} x \mathrm{~d} v \\
& =2^{\frac{1}{2}(k+1)-\delta} \operatorname{Vol}\left(\mathbb{S}^{k-2}\right) \mathrm{e}^{(k+1-2 \delta) h}\left(1+\mathcal{O}\left(\mathrm{e}^{-\frac{1}{2} h}\right)\right) \int_{0}^{\infty} J(\tau) \phi(\tau) \mathrm{d} \tau,
\end{aligned}
$$

avec

$$
J(\tau):=(\operatorname{sh} \tau) \int_{0}^{\infty}(1+(\operatorname{ch} \tau) \operatorname{ch} u)^{\frac{1}{2}(k-1)-\delta}(\operatorname{ch} u)^{\delta-k+1} \mathrm{~d} u .
$$

On constate que $J(\tau)=\mathcal{O}\left(\mathrm{e}^{\left(\frac{1}{2}(k+1)-\delta\right) \tau}\right)$, ce qui au vu de l'hypothèse d'intégrabilité faite sur $\phi^{\prime}$ justifie le remplacement de $\phi\left(\tau+\mathcal{O}\left(\mathrm{e}^{-\frac{1}{2} h}\right)\right)$ par $\phi(\tau)+\mathcal{O}\left(\mathrm{e}^{-\frac{1}{2} h}\right)$ dans les manipulations évoquées ci-dessus.

Cela justifie aussi, avec l'estimation de $|\nabla F|$ obtenue plus haut, qu'on puisse remplacer ci-dessus $F$ par le maximum local de $|\nabla F|$, pour obtenir

$$
\begin{aligned}
\left|\sum_{n \in \mathbb{Z}^{k}}\left(1_{\mathcal{E}_{h}^{\prime}} \times F\right)\left(\sum_{j=1}^{k} 2 n_{j} V_{j}\right)-\int_{\mathbb{R}^{k}}\left(1_{\mathcal{E}_{h}^{\prime}} \times F\right)\left(\sum_{j=1}^{k} 2 s_{j} V_{j}\right) \mathrm{d} s\right| \\
=\mathcal{O}\left(\mathrm{e}^{(k+1-2 \delta) h}\right) \times \mathcal{O}\left(\mathrm{e}^{-\frac{1}{2} h}\right)
\end{aligned}
$$

et donc

$$
\begin{aligned}
& \sum_{n \in \mathbb{Z}^{k}}\left(1_{\mathcal{E}_{h}^{\prime}} \times F\right)\left(\sum_{j=1}^{k} 2 n_{j} V_{j}\right) \\
& \quad=2^{\frac{1}{2}(k+1)-\delta} \frac{\operatorname{Vol}\left(\mathbb{S}^{k-2}\right)}{\operatorname{Covol}\left(\Gamma_{\infty}^{\prime \prime}\right)} \int_{0}^{\infty} J \phi \times \mathrm{e}^{(k+1-2 \delta) h}\left(1+\mathcal{O}\left(\mathrm{e}^{-\frac{1}{2} h}\right)\right),
\end{aligned}
$$

BULLETIN DE LA SOCIÉTÉ MATHÉMATIQUE DE FRANCE 
d'où au total

$$
\Sigma_{h}^{\prime}=2^{\frac{1}{2}(k+1)} \frac{\operatorname{Vol}\left(\mathbb{S}^{k-2}\right)}{\operatorname{Covol}\left(\Gamma_{\infty}\right)} \int_{0}^{\infty} J \phi \times \mathrm{e}^{(k+1-2 \delta) h}\left(1+\mathcal{O}\left(\mathrm{e}^{-\frac{1}{2} h}\right)\right) ;
$$

et puisque cette estimation est uniforme en $V_{-}, V_{+} \in \mathcal{D}$ :

$$
\Sigma_{h}=\lambda_{\mathcal{P}}^{2} 2^{\frac{1}{2}(k+1)} \frac{\operatorname{Vol}\left(\mathbb{S}^{k-2}\right)}{\operatorname{Covol}\left(\Gamma_{\infty}\right)} \int_{0}^{\infty} J \phi \times \mathrm{e}^{(k+1-2 \delta) h}\left(1+\mathcal{O}\left(\mathrm{e}^{-\frac{1}{2} h}\right)\right),
$$

ce qui est le résultat de l'énoncé dans le cas $k \neq 1$ et $\Gamma_{\infty}^{\prime} \not \subset \mathbb{C}^{d-1}$.

Envisageons ensuite le cas $\Gamma_{\infty}^{\prime \prime} \subset \mathbb{C}^{d-1}$ (i.e. $\left.\Gamma_{\infty}^{\prime} \subset \mathbb{C}^{d-1}\right)$; nous avons alors :

$$
\int_{\mathbb{R}^{k}}\left(1_{\mathcal{E}_{h}^{\prime}} \times F\right)\left(\sum_{j=1}^{k} 2 s_{j} V_{j}\right) \mathrm{d} s=\operatorname{Covol}\left(\Gamma_{\infty}^{\prime \prime}\right)^{-1} \int_{\mathbb{R}^{k}}\left(1_{\mathcal{E}_{h}^{\prime}} \times F\right)(x, 0) \mathrm{d} x
$$

et

$$
\begin{aligned}
& \int_{\mathbb{R}^{k}}\left(1_{\mathcal{E}_{h}^{\prime}} \times F\right)(x, 0) \mathrm{d} x \\
& =\operatorname{Vol}\left(\mathbb{S}^{k-1}\right) \mathrm{e}^{k h} \int_{0}^{\infty}\left(1_{\mathcal{E}_{h}^{\prime}} \times F\right)\left(\mathrm{e}^{h} r, 0\right) r^{k-1} \mathrm{~d} r \\
& =\operatorname{Vol}\left(\mathbb{S}^{k-1}\right) \mathrm{e}^{(k-2 \delta) h} \int_{0}^{\infty} 1_{\left\{4\left(1+\mathrm{e}^{-h}\right) \leq r^{2}\right\}} r^{k-1-2 \delta} \\
& \quad \times \phi\left(\log \left[r^{2}-2+r \sqrt{r^{2}-4}\right]-\log 2+\mathcal{O}\left(\mathrm{e}^{-\frac{1}{2} h}\right)\right) \mathrm{d} r \\
& =\operatorname{Vol}\left(\mathbb{S}^{k-1}\right) \int_{0}^{\infty}\left(2 \operatorname{ch} \frac{1}{2} t\right)^{k-1-2 \delta}\left(\operatorname{sh} \frac{1}{2} t\right) \phi(t) \mathrm{d} t \times \mathrm{e}^{(k-2 \delta) h}\left(1+\mathcal{O}\left(\mathrm{e}^{-\frac{1}{2} h}\right)\right) .
\end{aligned}
$$

De la même façon que pour le premier cas, ceci prouve l'énoncé dans le cas $\Gamma_{\infty}^{\prime} \subset \mathbb{C}^{d-1}$.

Envisageons ensuite le cas où $\Gamma_{\infty}^{\prime \prime} \subset \mathbb{R}\left(\right.$ i.e. $\left.\Gamma_{\infty}^{\prime} \subset \mathbb{R}\right)$ :

$$
\int_{\mathbb{R}}\left(1_{\mathcal{E}_{h}^{\prime}} \times F\right)\left(2 s V_{1}\right) \mathrm{d} s=\operatorname{Covol}\left(\Gamma_{\infty}^{\prime \prime}\right)^{-1} \int_{\mathbb{R}}\left(1_{\mathcal{E}_{h}^{\prime}} \times F\right)(0, v) \mathrm{d} v
$$

et

$$
\begin{aligned}
& \int_{\mathbb{R}}\left(1_{\mathcal{E}_{h}^{\prime}} \times F\right)(0, v) \mathrm{d} v=\mathrm{e}^{2 h} \int_{\mathbb{R}}\left(1_{\mathcal{E}_{h}^{\prime}} \times F\right)\left(0, \mathrm{e}^{2 h} v\right) \mathrm{d} v \\
& =2 \mathrm{e}^{2(1-\delta) h} \int_{0}^{\infty} 1_{\left\{2\left(1+\mathrm{e}^{-h}\right) \leq v\right\}} v^{-\delta} \phi\left(\log \left[\frac{1}{2}\left(v+\sqrt{v^{2}-4}\right)\right]+\mathcal{O}\left(\mathrm{e}^{-\frac{1}{2} h}\right)\right) \mathrm{d} v \\
& \times\left(1+\mathcal{O}\left(\mathrm{e}^{-h}\right)\right) \\
& =\mathrm{e}^{2(1-\delta) h} \cdot 2^{2-\delta} \int_{0}^{\infty}(\operatorname{ch} \tau)^{-\delta}(\operatorname{sh} \tau) \phi(\tau) \mathrm{d} \tau \times\left(1+\mathcal{O}\left(\mathrm{e}^{-\frac{1}{2} h}\right)\right) .
\end{aligned}
$$

Ceci prouve, de même que pour les cas précédents, l'énoncé dans le cas $\Gamma_{\infty}^{\prime} \subset \mathbb{R}$.

TOME $130-2002-\mathrm{N}^{\mathrm{O}} 3$ 
Envisageons enfin le cas où $k=1$ et $\Gamma_{\infty}^{\prime \prime} \not \subset \mathbb{C}^{d-1} \cup \mathbb{R}$ : nous pouvons considérer que $V_{1}=|\zeta|(1, \operatorname{tg} \theta)$, avec $0<\theta<\frac{1}{2} \pi$, et nous avons :

$$
\begin{gathered}
\int_{\mathbb{R}}\left(1_{\mathcal{E}_{h}^{\prime}} \times F\right)\left(2 s V_{1}\right) \mathrm{d} s=(2|\zeta|)^{-1} \mathrm{e}^{h} \int_{\mathbb{R}}\left(1_{\mathcal{E}_{h}^{\prime}} \times F\right)\left(\mathrm{e}^{h} x, \mathrm{e}^{h} x \operatorname{tg} \theta\right) \mathrm{d} x \\
=\frac{2 \mathrm{e}^{(1-2 \delta) h}\left(1+\mathcal{O}\left(\mathrm{e}^{-h}\right)\right)}{\operatorname{Covol}\left(\pi\left(\Gamma_{\infty}^{\prime \prime}\right)\right)} \int_{0}^{\infty} 1_{\left\{2\left(1+\mathrm{e}^{-h}\right) \leq x\right\}} x^{-2 \delta} \\
\times \phi\left(\log \left[\frac{x^{2}-2+\sqrt{x^{4}-4 x^{2}-\mathcal{O}\left(\mathrm{e}^{-2 h}\right)}}{2}\right]+\mathcal{O}\left(\mathrm{e}^{-\frac{1}{2} h}\right)\right) \mathrm{d} x \\
=\frac{2 \mathrm{e}^{(1-2 \delta) h}}{\operatorname{Covol}\left(\pi\left(\Gamma_{\infty}^{\prime \prime}\right)\right)} \int_{0}^{\infty}\left(2 \operatorname{ch} \frac{1}{2} t\right)^{-2 \delta}\left(\operatorname{sh} \frac{1}{2} t\right) \phi(t) \mathrm{d} t \times\left(1+\mathcal{O}\left(\mathrm{e}^{-\frac{1}{2} h}\right)\right),
\end{gathered}
$$

ce qui prouve, de même que pour les cas précédents, l'énoncé dans le dernier cas.

6.4. Masse asymptotique et temps de retour. - De même qu'aux sections 4 et 6 , les masses asymptotiques de la pointe et de la mesure de Palm se déduisent de la proposition 8 en choisissant respectivement $\phi=\operatorname{Id}$ et $\phi \equiv 1$.

Corollaire 5. - Nous avons lorsque $h \rightarrow \infty$ :

$$
\begin{gathered}
m\left(T^{1} \mathcal{P}_{h}\right)=K_{\mathcal{P}}^{\prime} \lambda_{\mathcal{P}}^{2} \operatorname{Covol}\left(\Gamma_{\xi}\right)^{-1} \mathrm{e}^{2\left(\delta_{\xi}-\delta\right) h}\left(1+\mathcal{O}\left(\mathrm{e}^{-\frac{1}{2} h}\right)\right), \\
\left|\chi_{h}\right|=K_{\mathcal{P}}^{\prime \prime} \lambda_{\mathcal{P}}^{2}\left(\delta-\delta_{\xi}\right)^{-1} \operatorname{Covol}\left(\Gamma_{\xi}\right)^{-1} \mathrm{e}^{2\left(\delta_{\xi}-\delta\right) h}\left(1+\mathcal{O}\left(\mathrm{e}^{-\frac{1}{2} h}\right)\right),
\end{gathered}
$$

où

1) $\operatorname{si} \Gamma_{\xi}^{\prime} \not \subset \mathbb{C}^{d-1}$ et $k \neq 1$ :

$K_{\mathcal{P}}^{\prime \prime}=2^{k+2-\delta} \operatorname{Vol}\left(\mathbb{S}^{k-2}\right) \int_{0}^{1}\left(1-t^{2}\right)^{\frac{1}{3}(k-3)}\left(1+t^{2}\right)^{\delta-k} \mathrm{~d} t$

$K_{\mathcal{P}}^{\prime}=2^{\frac{k+1}{2}} \operatorname{Vol}\left(\mathbb{S}^{k-2}\right)\left(\delta-\delta_{\xi}\right)^{-1} \int_{0}^{\infty} \int_{0}^{\infty}(1+(\operatorname{ch} \tau) \operatorname{ch} u)^{\frac{1}{2}(k+1)-\delta}(\operatorname{ch} u)^{\delta-k} \mathrm{~d} \tau \mathrm{d} u ;$

2) si $\Gamma_{\xi}^{\prime} \subset \mathbb{C}^{d-1}$ :

$$
K_{\mathcal{P}}^{\prime}=2^{\delta-1} \operatorname{Vol}\left(\mathbb{S}^{k-1}\right) \frac{\Gamma\left(\delta-\frac{1}{2} k\right)^{2}}{\Gamma(2 \delta-k+1)}, \quad K_{\mathcal{P}}^{\prime \prime}=2^{k-1-\delta} \operatorname{Vol}\left(\mathbb{S}^{k-1}\right)
$$

3) si $\Gamma_{\xi}^{\prime} \subset \mathbb{R}: K_{\mathcal{P}}^{\prime}=2^{\delta-1} \Gamma\left(\frac{1}{2}(\delta-1)\right)^{2} / \Gamma(\delta)$ et $K_{\mathcal{P}}^{\prime \prime}=4$;

4) si $\Gamma_{\xi}^{\prime} \not \subset \mathbb{C}^{d-1} \cup \mathbb{R}$ et $k=1$ :

$$
K_{\mathcal{P}}^{\prime}=\frac{2^{\delta} \operatorname{Covol}\left(\Gamma_{\xi}\right)}{\operatorname{Covol}\left(\pi\left(\Gamma_{\xi}\right)\right)} \cdot \frac{\Gamma\left(\delta-\frac{1}{2}\right)^{2}}{\Gamma(2 \delta)}, \quad K_{\mathcal{P}}^{\prime \prime}=\frac{2^{1-\delta} \operatorname{Covol}\left(\Gamma_{\xi}\right)}{\operatorname{Covol}\left(\pi\left(\Gamma_{\xi}\right)\right)} .
$$

De même qu'à la section 5 , nous en déduisons la version précisée de la proposition 4 :

BULLETIN DE LA SOCIÉtÉ MATHÉMATIQUE DE FRANCE 
Corollaire 6. - Nous avons m-presque sûrement :

$$
\lim _{N \rightarrow \infty} \frac{S_{h}^{2 N-1}}{N}=\lim _{N \rightarrow \infty} \frac{S_{h}^{2 N}}{N}=|m| \frac{\left(\delta-\delta_{\xi}\right)}{\lambda_{\mathcal{P}}^{2} K_{\mathcal{P}}^{\prime \prime}} \operatorname{Covol}\left(\Gamma_{\xi}\right) \mathrm{e}^{2\left(\delta-\delta_{\xi}\right) h}\left(1+\mathcal{O}\left(\mathrm{e}^{-\frac{1}{2} h}\right)\right) .
$$

Appliquant la proposition 8 avec $\phi(u)=\mathrm{e}^{\sqrt{-1} q u}$, comme en section 5 , nous obtenons la version précisée de la proposition 5 (avec les notations des propositions 7 et 8$)$ :

Corollaire 7. - Sous la probabilité $m /|m|$, la loi de la durée $\left(S_{h}^{2 j}-S_{h}^{2 j-1}\right)$ d'une excursion du flot géodésique dans $\mathcal{P}_{h}$ converge lorsque $h \rightarrow \infty$ vers la loi de densité proportionnelle $\grave{a}: J$ dans le cas $(1), t \mapsto\left(\operatorname{ch} \frac{1}{2} t\right)^{k-2 \delta-1} \operatorname{sh} \frac{1}{2} t$ dans les cas (2) et (4), $t \mapsto(\operatorname{ch} t)^{-\delta} \operatorname{sh} t$ dans le cas (3).

6.5. Enroulements asymptotiques. - Nous avons vu dans la section 6.2 que la géodésique $\overrightarrow{0(\zeta, v)}$ intersecte l'horocycle horizontal $\left\{t=\mathrm{e}^{2 h}\right\}$ lorsque $N^{4} /\left(N^{2}+|\zeta|^{2}\right) \geq 2 \mathrm{e}^{2 h}$, en les deux points de coordonnées horocycliques $\left(\zeta_{h}^{-}, v_{h}^{-}, \mathrm{e}^{2 h}\right)$ et $\left(\zeta_{h}^{+}, v_{h}^{+}, \mathrm{e}^{2 h}\right)$, déterminés en coordonnées projectives par

$$
P\left(2 \mathrm{e}^{2 s \pm} \zeta,\left(|\zeta|^{2}-i v\right) \mathrm{e}^{2 s \pm}, 2\left(|\zeta|^{2}+i v\right)^{-1}+2 \mathrm{e}^{2 s \pm}\right)
$$

où

$$
\mathrm{e}^{2 s \pm}=\frac{N^{4}-2|\zeta|^{2} \mathrm{e}^{2 h} \pm \sqrt{N^{8}-4 N^{4}|\zeta|^{2} \mathrm{e}^{2 h}-4 v^{2} \mathrm{e}^{4 h}}}{2 N^{4} \mathrm{e}^{2 h}} .
$$

L'enroulement effectué dans $\mathcal{P}_{h}$ par la géodésique $\overrightarrow{0(\zeta, v)}$ est déterminé par l'élément

$$
\left(\zeta_{h}, v_{h}\right):=\left(\zeta_{h}^{+}, v_{h}^{+}\right) \cdot\left(\zeta_{h}^{-}, v_{h}^{-}\right)^{-1}=\left(\zeta_{h}^{+}-\zeta_{h}^{-}, v_{h}^{+}-v_{h}^{-}-2 \operatorname{Im}\left(\zeta_{h}^{+} \cdot \overline{\zeta_{h}^{-}}\right)\right) \in \mathcal{H}_{d},
$$

étant donné que dans le modèle fixé ci-dessus pour $\mathbb{H}_{\mathbb{C}}^{d}$ les isométries opèrent à gauche. Le calcul donne :

$$
\begin{aligned}
\zeta_{h} & =\left(\frac{\mathrm{e}^{2 s+}}{1+\left(|\zeta|^{2}+i v\right) \mathrm{e}^{2 s+}}-\frac{\mathrm{e}^{2 s-}}{1+\left(|\zeta|^{2}+i v\right) \mathrm{e}^{2 s-}}\right)\left(|\zeta|^{2}+i v\right) \zeta \\
& =N^{-2} \sqrt{N^{4}-4|\zeta|^{2} \mathrm{e}^{2 h}-4 v^{2} N^{-4} \mathrm{e}^{4 h}} \times \zeta
\end{aligned}
$$

et

$$
\begin{aligned}
v_{h}= & -2 \operatorname{Im}\left(\frac{\left(|\zeta|^{2}-i v\right) \mathrm{e}^{2 s+}}{2\left(|\zeta|^{2}+i v\right)^{-1}+2 \mathrm{e}^{2 s+}}\right)+2 \operatorname{Im}\left(\frac{\left(|\zeta|^{2}-i v\right) \mathrm{e}^{2 s-}}{2\left(|\zeta|^{2}+i v\right)^{-1}+2 \mathrm{e}^{2 s-}}\right) \\
& -2 \operatorname{Im}\left(\frac{4|\zeta|^{2} \mathrm{e}^{2 s+} \mathrm{e}^{2 s-}}{\left(2\left(|\zeta|^{2}+i v\right)^{-1}+2 \mathrm{e}^{2 s+}\right)\left(2\left(|\zeta|^{2}+i v\right)^{-1}+2 \mathrm{e}^{2 s-}\right)}\right) \\
= & N^{-2} \sqrt{N^{4}-4|\zeta|^{2} \mathrm{e}^{2 h}-4 v^{2} N^{-4} \mathrm{e}^{4 h}} \times\left(1+2|\zeta|^{2} N^{-4} \mathrm{e}^{2 h}\right) v .
\end{aligned}
$$

TOME $130-2002-\mathrm{N}^{\mathrm{O}} 3$ 
À $\gamma \in \Gamma$ et $V_{-}, V_{+} \in \mathcal{H}_{d}$, associons lorsqu'elles existent les deux intersections $V_{h}^{-}, V_{h}^{+}$de la géodésique $\overrightarrow{V_{-} \gamma V_{+}}$avec l'horocycle horizontal $\left\{t=\mathrm{e}^{2 h}\right\}$, puis

$$
\left(\zeta_{h}, v_{h}\right)=\left(\zeta_{h}, v_{h}\right)\left(V_{-}, \gamma V_{+}\right):=V_{h}^{+} \cdot\left(V_{h}^{-}\right)^{-1} \in \mathcal{H}_{d} .
$$

C'est $\left(\zeta_{h}, v_{h}\right)\left(V_{-}, \gamma V_{+}\right)$qui détermine l'enroulement (non normalisé) effectué lors d'une excursion dans $\mathcal{P}_{h}$ par la projection sur $\mathcal{M}$ de la géodésique $\overrightarrow{V_{-} \gamma V_{+}}$.

Les distances horocycliques, dans $\left\{t=\mathrm{e}^{2 h}\right\}$, de $\left(0,0, \mathrm{e}^{2 h}\right) \grave{\mathrm{a}}\left(\zeta_{h}, 0, \mathrm{e}^{2 h}\right)$ et de $\left(0,0, \mathrm{e}^{2 h}\right)$ à $\left(0, v_{h}, \mathrm{e}^{2 h}\right)$ étant respectivement $\mathrm{e}^{-h}\left|\zeta_{h}\right|$ et $\frac{1}{2} \mathrm{e}^{-2 h}\left|v_{h}\right|$, il convient de normaliser $\left(\zeta_{h}, v_{h}\right)$ en $\left(\mathrm{e}^{-h} \zeta_{h}, \frac{1}{2} \mathrm{e}^{-2 h} v_{h}\right)$.

La loi de l'enroulement d'une excursion géodésique dans $\mathcal{P}_{h}$ est ainsi donnée, de même qu'au début de la preuve du théorème 1 , par $\Theta_{h}^{F} /\left|\chi_{h}\right|$, où $F$ est une fonction-test et

$$
\begin{aligned}
& \Theta_{h}^{F}:=\int_{\mathcal{D}^{2}} \sum_{\gamma \in \Gamma_{\infty}} 1_{\mathcal{E}_{h}}\left(\left(V_{-}\right)^{-1} \cdot \gamma V_{+}\right) \\
& \quad \times F\left[\left(\frac{\zeta_{h}}{\mathrm{e}^{h}}, \frac{v_{h}}{2 \mathrm{e}^{2 h}}\right)\left(V_{-}, \gamma V_{+}\right)\right] d_{\xi}\left(V_{-}, \gamma V_{+}\right)^{-2 \delta} \frac{\mathrm{d} \mu_{\xi}\left(V_{-}\right) \mathrm{d} \mu_{\xi}\left(V_{+}\right)}{\left|\chi_{h}\right|} .
\end{aligned}
$$

$V_{-}, V_{+}$variant dans le compact fixe $\overline{\mathcal{D}}$ de $\mathcal{H}_{d}$, notons $\left(\zeta_{h}^{0}, v_{h}^{0}\right)(\gamma):=$ $\left(\zeta_{h}, v_{h}\right)\left(V_{-}, \gamma V_{+}\right)$, de sorte que nous avons :

$$
\begin{aligned}
& \Theta_{h}^{F}=\frac{\left[\Gamma_{\infty}: \Gamma_{\infty}^{\prime \prime}\right] \lambda_{\mathcal{P}}^{2}}{\left|\chi_{h}\right|} \sum_{\gamma \in \Gamma_{\infty}^{\prime \prime}} 1_{\mathcal{E}_{h}}(\gamma) \times F\left[\left(\frac{\zeta_{h}^{0}}{\mathrm{e}^{h}}, \frac{v_{h}^{0}}{2 \mathrm{e}^{2 h}}\right)\left(V_{-}, \gamma V_{+}\right)\right]\left(d_{\xi}(0, \gamma)\right)^{-2 \delta} \times\left(1+\mathcal{O}\left(\mathrm{e}^{-h}\right)\right) \\
&=2^{\delta} \frac{\left[\Gamma_{\infty}: \Gamma_{\infty}^{\prime \prime}\right] \lambda_{\mathcal{P}}^{2}}{\left|\chi_{h}\right|} \sum_{n \in \mathbb{Z}^{k}}\left(1_{\mathcal{E}_{h}} \times F\left[\frac{\zeta_{h}^{0}}{\left.\mathrm{e}^{h}, \frac{v_{h}^{0}}{2 \mathrm{e}^{2 h}}\right]} N^{-2 \delta}\right)\right. \\
& \times\left(\sum_{j=1}^{k} 2 n_{j} V_{j}\right)\left(1+\mathcal{O}\left(\mathrm{e}^{-h}\right)\right) .
\end{aligned}
$$

Notons par commodité

$$
R_{h}=R_{h}(\zeta, v):=\sqrt{N^{4}-4|\zeta|^{2} \mathrm{e}^{2 h}-4 v^{2} N^{-4} \mathrm{e}^{4 h}} .
$$

De même qu'aux sections 6.2 et 6.3 , la perturbation de $(\zeta, v)$ (par $V_{+}, V_{-}$variant dans le compact fixe $\overline{\mathcal{D}}$ de $\mathcal{H}_{d}$ ) conduit pour les quantités $\zeta_{h}^{0}, v_{h}^{0}, R_{h}^{0}$ perturbées de $\zeta_{h}, v_{h}, R_{h}$ aux expressions suivantes sur $\mathcal{E}_{h}^{\prime}$ (voir la section 6.2) :

$$
\begin{aligned}
& R_{h}^{0}=R_{h}+\mathcal{O}\left(\mathrm{e}^{\frac{3}{2} h}\right), \zeta_{h}^{0}=N^{-2}(1+\mathcal{O}(1 / N))\left[R_{h}+\mathcal{O}\left(\mathrm{e}^{\frac{3}{2} h}\right)\right](\zeta+\mathcal{O}(1)), \\
& v_{h}^{0}=N^{-2}(1+\mathcal{O}(1 / N))\left[R_{h}+\mathcal{O}\left(\mathrm{e}^{\frac{3}{2} h}\right)\right]\left(1+2|\zeta|^{2} N^{-4} \mathrm{e}^{2 h}(1+\mathcal{O}(1 /|\zeta|))\right) \\
& \times(v+\mathcal{O}(N)) .
\end{aligned}
$$

La contribution relative à $\mathcal{E}_{h}^{\prime \prime}$ se contrôle de la même façon que pour la proposition 8. Nous pouvons donc nous restreindre de $\mathcal{E}_{h}$ à $\mathcal{E}_{h}^{\prime}$, et donc utiliser 
les expressions ci-dessus pour $\zeta_{h}^{0}, v_{h}^{0}$. Toujours comme pour la proposition 8 , et comme pour le théorème 1 , la méthode des rectangles permet de substituer une intégrale à la série intervenant dans $\Theta_{h}^{F}$.

Plaçons-nous d'abord dans le cas (1) où $\Gamma_{\infty}^{\prime} \not \subset \mathbb{C}^{d-1}$ et $k \neq 1$. Alors

$$
\begin{aligned}
\operatorname{Covol}\left(\Gamma_{\infty}^{\prime \prime}\right) \sum_{n \in \mathbb{Z}^{k}}\left(1_{\mathcal{E}_{h}^{\prime}} \times F\left[\frac{\zeta_{h}^{0}}{\mathrm{e}^{h}}, \frac{v_{h}^{0}}{2 \mathrm{e}^{2 h}}\right] N^{-2 \delta}\right)\left(\sum_{j=1}^{k} 2 n_{j} V_{j}\right) \\
\sim \int_{\mathbb{R}^{k-1} \times \mathbb{R}} 1_{\mathcal{E}_{h}^{\prime} \times F\left[\frac{\zeta_{h}^{0}}{\mathrm{e}^{h}}, \frac{v_{h}^{0}}{2 \mathrm{e}^{2 h}}\right] N^{-2 \delta}} 1_{\left\{2 \mathrm{e}^{2 h}\left(1+\mathrm{e}^{-h}\right) \leq|x|^{4}+v^{2} / \sqrt{|x|^{4}+v^{2}}+|x|^{2}\right\}} \times\left(|x|^{4}+v^{2}\right)^{-\frac{1}{2} \delta} \\
\left.=\int_{\mathbb{R}^{k-1} \times \mathbb{R}} 1 \times \frac{R_{h}+\mathcal{O}\left(\mathrm{e}^{\frac{3}{2} h}\right)}{\sqrt{|x|^{4}+v^{2}}}\right) \mathrm{d} x \mathrm{~d} v \\
\quad \times F\left(\left(\frac{x+\mathcal{O}(1)}{\mathrm{e}^{h}}, \frac{v+\mathcal{O}\left(\left[|x|^{4}+v^{2}\right]^{\frac{1}{4}}\right)}{|x|^{4}+v^{2 h}\left(1+\mathcal{O}\left(|x|^{-1}\right)\right)}\right]\right)
\end{aligned}
$$

qui, après application du théorème de convergence dominée et quelques changements de variables, est équivalent à :

$$
\begin{array}{r}
\sim \int_{\mathbb{R}^{k-1} \times \mathbb{R}} 1_{\left\{2 \leq|x|^{2} / \sqrt{1+v^{2}}+1\right\}} F( \\
\left.\quad\left(\frac{x}{\sqrt{1+v^{2}}}, \frac{v\left(|x|^{2}+2\right)}{2\left(1+v^{2}\right)}\right) \frac{\left(|x|^{4}-4|x|^{2}-4 v^{2}\right)^{\frac{1}{2}}}{|x|^{2}}\right) \\
\times \mathrm{e}^{(k+1-2 \delta) h} \int_{\mathbb{S}^{k-2} \times \mathbb{R}_{+} \times \mathbb{R}} F\left(\left(\frac{1}{2} \sigma \sqrt{1+\operatorname{ch} u \operatorname{ch} \tau}, \operatorname{th} \tau(2+\operatorname{ch} u \operatorname{ch} \tau)\right) \frac{2 \operatorname{sh} u}{1+\operatorname{ch} u \operatorname{ch} \tau}\right) \\
\times 2^{\frac{1}{2}(k-1)-\delta}(1+\operatorname{ch} u \operatorname{ch} \tau)^{\frac{1}{2}(k-1)-\delta}(\operatorname{ch} \tau)^{\delta-k+1} \operatorname{sh} u \mathrm{~d} \sigma \mathrm{d} u \mathrm{~d} \tau .
\end{array}
$$

Notons

$$
R(u, \tau):=\frac{\sqrt{2} \operatorname{sh} u}{\sqrt{1+\operatorname{ch} u \operatorname{ch} \tau}}, \quad V(u, \tau):=2 \operatorname{sh} u \operatorname{th} \tau \times \frac{2+\operatorname{ch} u \operatorname{ch} \tau}{1+\operatorname{ch} u \operatorname{ch} \tau} .
$$

Rassemblant ce qui précède et le corollaire 5, nous obtenons :

$\lim _{h \rightarrow \infty} \frac{\Theta_{h}^{F}}{\left|\chi_{h}\right|}=2^{\frac{1}{2}(k-3)} \frac{2 \delta-k-1}{K_{\mathcal{P}}^{\prime \prime}} \int_{\mathbb{S}^{k-2} \times \mathbb{R}_{+} \times \mathbb{R}} F(\sigma R(u, \tau), V(u, \tau)) \varphi(u, \tau) \mathrm{d} \sigma \mathrm{d} u \mathrm{~d} \tau$,

où $\varphi(u, \tau):=(1+\operatorname{ch} u \operatorname{ch} \tau)^{\frac{1}{2}(k-1)-\delta}(\operatorname{ch} \tau)^{\delta-k+1} \operatorname{sh} u$, ce qui détermine la loi limite sous $m$ de l'enroulement d'une excursion géodésique dans $\mathcal{P}_{h}$, dans le cas (1).

TOME $130-2002-\mathrm{N}^{\mathrm{O}} 3$ 
Passons au cas (2), lorsque $\Gamma_{\infty}^{\prime} \subset \mathbb{C}^{d-1}$ :

$$
\begin{aligned}
& \operatorname{Covol}\left(\Gamma_{\infty}^{\prime \prime}\right) \sum_{n \in \mathbb{Z}^{k}}\left(1_{\mathcal{E}_{h}^{\prime}} \times F\left[\frac{\zeta_{h}^{0}}{\mathrm{e}^{h}}, \frac{v_{h}^{0}}{2 \mathrm{e}^{2 h}}\right] N^{-2 \delta}\right)\left(\sum_{j=1}^{k} 2 n_{j} V_{j}\right) \\
& \sim \int_{\mathbb{R}^{k}} 1_{\mathcal{E}_{h}^{\prime}} \times F\left[\frac{\zeta_{h}^{0}}{\mathrm{e}^{h}}, \frac{v_{h}^{0}}{2 \mathrm{e}^{2 h}}\right] N^{-2 \delta} \\
& =\int_{\mathbb{R}^{k}} 1_{\left\{2 \mathrm{e}^{2 h}\left(1+\mathrm{e}^{-h}\right) \leq|x|^{2} / 2\right\}} \times|x|^{-2 \delta} \\
& \times F\left(\left(\mathrm{e}^{-h}(x+\mathcal{O}(1)), \mathrm{e}^{-2 h} \mathcal{O}(|x|)\left[\frac{1}{2}-\frac{\mathrm{e}^{2 h}\left(1+\mathcal{O}\left(|x|^{-1}\right)\right)}{|x|^{2}}\right]\right)\right. \\
& \left.\times \frac{\sqrt{|x|^{4}-4|x|^{2} \mathrm{e}^{2 h}}+\mathcal{O}\left(\mathrm{e}^{\frac{3}{2} h}\right)}{|x|^{2}}\right) \mathrm{d} x \\
& \sim \mathrm{e}^{(k-2 \delta) h} \int_{\mathbb{S}^{k-1} \times \mathbb{R}_{+}} F(\sigma R, 0)\left(R^{2}+4\right)^{\frac{1}{2} k-\delta-1} R \mathrm{~d} R \mathrm{~d} \sigma .
\end{aligned}
$$

Avec de nouveau l'aide du corollaire 5 , nous obtenons donc :

$$
\lim _{h \rightarrow \infty} \frac{\Theta_{h}^{F}}{\left|\chi_{h}\right|}=2^{2 \delta-k} \frac{2 \delta-k}{\operatorname{Vol}\left(\mathbb{S}^{k-1}\right)} \int_{\mathbb{S}^{k-1} \times \mathbb{R}_{+}} F(\sigma r, 0)\left(r^{2}+4\right)^{\frac{1}{2} k-\delta-1} r \mathrm{~d} r \mathrm{~d} \sigma,
$$

ce qui explicite la loi limite sous $m$ de l'enroulement d'une excursion géodésique dans $\mathcal{P}_{h}$, dans le cas $(2)$.

Passons au cas (3), lorsque $\Gamma_{\infty}^{\prime} \subset \mathbb{R}$ :

$$
\begin{aligned}
\operatorname{Covol}\left(\Gamma_{\infty}^{\prime \prime}\right) \sum_{n \in \mathbb{Z}}\left(1_{\mathcal{E}_{h}^{\prime}} \times F\left[\frac{\zeta_{h}^{0}}{\mathrm{e}^{h}}, \frac{v_{h}^{0}}{2 \mathrm{e}^{2 h}}\right] \times N^{-2 \delta}\right)\left(2 n V_{1}\right) \\
\quad \sim \int_{\mathbb{R}} 1_{\mathcal{E}_{h}^{\prime}} \times F\left[\frac{\zeta_{h}^{0}}{\mathrm{e}^{h}}, \frac{v_{h}^{0}}{2 \mathrm{e}^{2 h}}\right] N^{-2 \delta} \\
=\int_{\mathbb{R}} 1_{\left\{2 \mathrm{e}^{2 h}\left(1+\mathrm{e}^{-h}\right) \leq|v|\right\}} F\left(\left(\mathcal{O}\left(\mathrm{e}^{-h}\right), \mathrm{e}^{-2 h}\left(\frac{1}{2} v+\mathcal{O}(\sqrt{|v|})\right)\right)\right. \\
\left.\quad \times \frac{\sqrt{v^{2}-4 \mathrm{e}^{4 h}}+\mathcal{O}\left(\mathrm{e}^{\frac{3}{2} h}\right)}{|v|}\right)|v|^{-\delta} \mathrm{d} v \\
\sim \mathrm{e}^{2(1-\delta) h} \int_{\mathbb{R}} 1_{\{2 \leq|v|\}} F\left(0, \frac{1}{2} v \sqrt{1-4 v^{-2}}\right)|v|^{-\delta} \mathrm{d} v \\
=\frac{\mathrm{e}^{2(1-\delta) h}}{2^{\delta-1}} \int_{\mathbb{R}} F(0, v)\left(v^{2}+1\right)^{-\frac{1}{2}(\delta+1)}|v| \mathrm{d} v,
\end{aligned}
$$

d'où via le corollaire 5 :

$$
\lim _{h \rightarrow \infty} \frac{\Theta_{h}^{F}}{\left|\chi_{h}\right|}=\frac{\delta-1}{2} \int_{\mathbb{R}} F(0, v)\left(v^{2}+1\right)^{-\frac{1}{2}(\delta+1)}|v| \mathrm{d} v
$$

BULLETIN DE LA SOCIÉTÉ MATHÉMATIQUE DE FRANCE 
ce qui explicite la loi limite sous $m$ de l'enroulement d'une excursion géodésique dans $\mathcal{P}_{h}$, dans le cas $(3)$.

Passons au cas (4), lorsque $\Gamma_{\infty}^{\prime} \not \subset \mathbb{C}^{d-1} \cup \mathbb{R}$ et $k=1: V_{1}=|\zeta|(1, \operatorname{tg} \theta)$ et

$$
\begin{aligned}
\sum_{n \in \mathbb{Z}} & \left(1_{\mathcal{E}_{h}^{\prime}} \times F\left[\frac{\zeta_{h}^{0}}{\mathrm{e}^{h}}, \frac{v_{h}^{0}}{2 \mathrm{e}^{2 h}}\right] \times N^{-2 \delta}\right)\left(2 n V_{1}\right) \\
\sim & (2|\zeta|)^{-1} \int_{\mathbb{R}}\left(1_{\mathcal{E}_{h}^{\prime}} \times F\left[\frac{\zeta_{h}^{0}}{\mathrm{e}^{h}}, \frac{v_{h}^{0}}{2 \mathrm{e}^{2 h}}\right] N^{-2 \delta}\right)(s, s \operatorname{tg} \theta) \mathrm{d} s \\
= & (2|\zeta|)^{-1} \int_{\mathbb{R}} 1_{\left\{2 \mathrm{e}^{2 h}\left(1+\mathrm{e}^{-h}\right) \leq s^{2}+\operatorname{tg}^{2} \theta / \sqrt{1+s^{-2} \operatorname{tg}^{2} \theta}+1\right\}} \times\left(s^{4}+s^{2} \operatorname{tg}^{2} \theta\right)^{-\frac{1}{2} \delta} \\
& \times F\left(\left(\frac{s+\mathcal{O}(1)}{\mathrm{e}^{h}}, \frac{s \operatorname{tg} \theta+\mathcal{O}\left(\left[s^{4}+s^{2} \operatorname{tg}^{2} \theta\right]^{\frac{1}{4}}\right)}{2 \mathrm{e}^{2 h}}\left[1-\frac{2 s^{2} \mathrm{e}^{2 h}\left(1+\mathcal{O}\left(s^{-1}\right)\right)}{s^{4}+s^{2} \operatorname{tg}^{2} \theta}\right]\right)\right. \\
\sim & (2|\zeta|)^{-1} \mathrm{e}^{2(1-\delta) h} \int_{\mathbb{R}} F(x, 0)\left(x^{2}+4\right)^{-\delta-\frac{1}{2}}|x| \mathrm{d} x,
\end{aligned}
$$

d'où via le corollaire 5 :

$$
\lim _{h \rightarrow \infty} \frac{\Theta_{h}^{F}}{\left|\chi_{h}\right|}=2^{2 \delta-2}(2 \delta-1) \int_{\mathbb{R}} F(x, 0)\left(x^{2}+4\right)^{-\delta-\frac{1}{2}}|x| \mathrm{d} x,
$$

ce qui explicite la loi limite sous $m$ de l'enroulement d'une excursion géodésique dans $\mathcal{P}_{h}$, dans le cas (4). Remarquons que ce dernier cas se rattache au cas (2), qui lui-même rejoint le cas hyperbolique réel.

Nous venons d'établir le résultat suivant.

ThÉORÈme 2. - Sous la probabilité invariante $m /|m|$, la loi de l'enroulement $\left(\zeta_{h} / \mathrm{e}^{h}, v_{h} / 2 \mathrm{e}^{2 h}\right)$ de la première excursion complète accomplie par le flot géodésique dans $\mathcal{P}_{h}$ converge lorsque $h \rightarrow+\infty$ vers la loi

1) si $\Gamma_{\xi}^{\prime} \not \subset \mathbb{C}^{d-1}$ et $k \neq 1$ : de $(\sigma R, V)$ sur $\mathbb{R}^{k-1} \times \mathbb{R}$, où $\sigma$ est uniforme sur $\mathbb{S}^{k-2}$ et indépendante de $(R, V)$, et où

$$
R:=\frac{\sqrt{2} \operatorname{sh} U}{\sqrt{1+\operatorname{ch} U \operatorname{ch} T}}, \quad V:=2 \operatorname{sh} U \operatorname{th} T \times \frac{2+\operatorname{ch} U \operatorname{ch} T}{1+\operatorname{ch} U \operatorname{ch} T},
$$

$(U, T)$ ayant sur $\mathbb{R}_{+} \times \mathbb{R}$ la densité

$$
(u, \tau) \longmapsto c(1+\operatorname{ch} u \operatorname{ch} \tau)^{\frac{1}{2}(k-1)-\delta}(\operatorname{ch} \tau)^{\delta-k+1} \operatorname{sh} u ;
$$

2) et 4) si $\Gamma_{\xi}^{\prime} \subset \mathbb{C}^{d-1}$ ou si $\Gamma_{\xi}^{\prime} \not \subset \mathbb{R}$ et $k=1$ : qui a pour densité sur $\mathbb{R}^{k}$

$$
x \longmapsto \frac{2^{2 \delta-k}(2 \delta-k)}{\operatorname{Vol}\left(\mathbb{S}^{k-1}\right)} \times\left(|x|^{2}+4\right)^{\frac{1}{2} k-\delta-1}|x|^{2-k},
$$

la deuxième composante convergeant vers 0 ;

TOME $130-2002-\mathrm{N}^{\mathrm{O}} 3$ 
3) si $\Gamma_{\xi}^{\prime} \subset \mathbb{R}:$ de densité

$$
v \longmapsto \frac{1}{2}(\delta-1) \times\left(v^{2}+1\right)^{-\frac{1}{2}(\delta+1)}|v|
$$

sur $\mathbb{R}$, la première composante convergeant vers 0 .

\section{BIBLIOGRAPHIE}

[1] Apanasov (B.) \& XIE (X.) - Geometrically finite complex hyperbolic manifolds, Intern. J. of Math., t. 8 (1997), no. 6, pp. 703-757.

[2] Bourdon (M.) - Structure conforme au bord et flot géodésique d'un CAT(-1)-espace, L'Ens. Math., t. 41 (1995), pp. 63-102.

[3] Bowditch (B.H.) - Geometrical finiteness with variable negative curvature, Duke Math. J., t. 77 (1995), no. 1, pp. 229-274.

[4] Corlette (K.) \& Iozzi (A.) - Limit sets of discrete groups of isometries of exotic hyperbolic spaces, Trans. AMS, t. 351 (1999), no.4, pp. $1507-$ 1530.

[5] Dal'bo (F.), Otal (J.-P.) \& Peigné (M.) - Séries de Poincaré des groupes géométriquement finis, Israël J. Math., t. 118 (2000), pp. 109-124.

[6] de Sam Lazaro (J.) \& Meyer (P.-A.) - Questions de théorie des flots, in Séminaire de Probabilités IX (Meyer (P.-A.), éd.), Lecture Notes, vol. 465, Springer, 1975.

[7] Enriquez (N.), Franchi (J.) \& Le Jan (Y.) - Stable windings on hyperbolic surfaces, Prob. Th. Rel. Fields, t. 119 (2001), pp. 213-255.

[8] FELleR (W.) - An introduction to probability theory and its applications, II, J. Wiley, New-York, 1966, 1971.

[9] Goldman (W.M.) - Complex hyperbolic geometry, Oxford Univ. Press, 1999.

[10] Guivarc'H (Y.) \& LE JAN (Y.) - Asymptotic winding of the geodesic flow on modular surfaces and continued fractions, Ann. scient. Éc. Norm. Sup. $4^{\text {e }}$ série, t. 26 (1993), no. 1, pp. 23-50.

[11] Hersonsky (S.) \& Paulin (F.) - On the volumes of complex hyperbolic manifolds, Duke Math. J., t. 84 (1996), no. 3, pp. 719-737.

[12] On the rigidity of discrete isometry groups of negatively curved spaces, Comment. Math. Helv., t. 72 (1997), pp. 349-388.

[13] _ Diophantine approximation in negatively curved manifolds and in the Heisenberg group, version préliminaire, 2001.

[14] Kaimanovich (V.) - Invariant measures of the geodesic flow and measures at infinity of negatively curved manifolds, Ann. IHP Phys. Th., t. 53 (1990), no. 4, pp. 361-393. 
[15] PATterson (S.J.) - Lectures on measures on limit sets of Kleinian groups, in Analytical and geometrical aspects of hyperbolic space (Epstein (D.), éd.), London Math. Society, Lecture Note Series, vol. 111, Cambridge University Press, 1987, pp. 281-323.

[16] Roblin (T.) - Sur la fonction orbitale des groupes discrets en courbure négative, Ann. Inst. Fourier, t. 52 (2002), no. 1, pp. 145-151.

[17] Sullivan (D.) - The density at infinity of a discrete group of hyperbolic motions, Publ. Math. IHES, t. 50 (1979), pp. 171-209.

[18] _ Entropy, Hausdorff measures old and new, and limit sets of geometrically finite Kleinian groups, Acta Math., t. 153 (1984), pp. 259-277. 